\title{
On the asymptotic behavior of holomorphic isometries of the Poincaré disk into \\ bounded symmetric domains
}

\author{
Ngaiming Mok*
}

\begin{abstract}
In this article we study holomorphic isometries of the Poincaré disk into bounded symmetric domains. Earlier we solved the problem of analytic continuation of germs of holomorphic maps between bounded domains which are isometries up to normalizing constants with respect to the Bergman metric, showing in particular that the graph $V_{0}$ of any germ of holomorphic isometry of the Poincaré disk $\Delta$ into an irreducible bounded symmetric domain $\Omega \Subset \mathbb{C}^{N}$ in its HarishChandra realization must extend to an affine-algebraic subvariety $V \subset \mathbb{C} \times \mathbb{C}^{N}=\mathbb{C}^{N+1}$, and that the irreducible component of $V \cap(\Delta \times \Omega)$ containing $V_{0}$ is the graph of a proper holomorphic isometric embedding $F: \Delta \rightarrow \Omega$. In this article we study holomorphic isometric embeddings which are asymptotically geodesic at a general boundary point $b \in \partial \Delta$. Starting with the structural equation for holomorphic isometries arising from the Gauss equation, we obtain by covariant differentiation an identity relating certain holomorphic bisectional curvatures to the boundary behavior of the second fundamental form $\sigma$ of the holomorphic isometric embedding. Using the nonpositivity of holomorphic bisectional curvatures on a bounded symmetric domain, we prove that $\|\sigma\|$ must vanish at a general boundary point either to the order 1 or to the order $\frac{1}{2}$, called a holomorphic isometry of the first resp. second kind. We deal with special cases of non-standard holomorphic isometric embeddings of such maps, showing that they must be asymptotically totally geodesic at a general boundary point and in fact of the first kind whenever the target domain is a Cartesian product of complex unit balls. We also study the boundary behavior of an example of holomorphic isometric embedding from the Poincaré disk into a Siegel upper half-plane by an explicit determination of the boundary behavior of holomorphic sectional curvatures in the directions tangent to the embedded Poincaré disk, showing that the map is indeed asymptotically totally geodesic at a general boundary point and of the first kind. For the metric computation we make use of formulas for symplectic geometry on Siegel upper half-planes.
\end{abstract}

Key words: holomorphic isometry, Bergman metric, Poincaré disk, analytic continuation, bounded symmetric domain, asymptotic geodesy, second fundamental form, Siegel upper half-plane, symplectic geometry

2000 Mathematics Subject Classification: 53B25, 53C35, 53C55

By the Poincaré disk we will mean the unit disk equipped with the Poincaré metric of constant negative Gaussian curvature $-\kappa$ for any positive real number $\kappa$. In what follows we will discuss holomorphic isometries of the Poincaré disk into bounded symmetric domains. The study of holomorphic isometries between Kähler manifolds started with the works of Bochner and Calabi. Especially, Calabi $[\mathrm{Ca}]$ proved theorems on rigidity and analytic continuation for holomorphic isometries of Kähler manifolds into holomorphic space forms such as the (possibly infinite-dimensional) projective space equipped with the Fubini-Study metric. More recently, motivated by a problem in Arithmetic Geometry, Clozel-Ullmo [CU] studied the question of characterizing holomorphic isometries of the Poincaré disk into the polydisk equipped with the Bergman metric, proving in particular the algebraic extension of the graph

*Partially supported by a research grant of the Research Grants Council, Hong Kong. 
of any such a germ of holomorphic isometry as an affine algebraic variety under an integrality condition on the normalizing constant. In Mok [Mk4] we studied the problem of analytic continuation of germs of holomorphic isometries up to normalizing constants with respect to the Bergman metric between bounded domains, proving in general interior extension results and furthermore boundary extension results when the Bergman kernels extend real-analytically across the boundary in some precise sense. In the special case of an irreducible bounded symmetric domain in its Harish-Chandra realization, the Bergman kernel $K(z, w)$ extends as a rational function in $(z, \bar{w})$. As a consequence, the graph $V_{0}$ of any germ of holomorphic isometry of the Poincaré disk $\Delta$ into an irreducible bounded symmetric domain $\Omega \Subset \mathbb{C}^{N}$ extends to an affine-algebraic subvariety $V \subset \mathbb{C} \times \mathbb{C}^{N}=\mathbb{C}^{N+1}$. Our results in [Mk4] also show that the irreducible component of $V \cap(\Delta \times \Omega)$ containing $V_{0}$ is the graph of a proper holomorphic isometric embedding $f$ of the Poincaré disk into $\Omega$. As a consequence, at all but a finite number of the boundary points on the unit circle $f$ extends holomorphically to a neighborhood of the boundary point.

In this article by a non-standard holomorphic isometric embedding of the Poincaré disk we mean a (necessarily proper) holomorphic isometric embedding $f: \Delta \rightarrow \Omega$ of the Poincaré disk into a bounded symmetric domain. Examples of non-standard holomorphic isometric embeddings of the Poincaré disk were constructed in Mok [Mk4]. The simplest of these are mappings into polydisks which in terms of unbounded realizations are given by the $p$-th root maps from the upper half-plane to a Cartesian product of upper half-planes (cf. (2.1) here). By various ways of compositions they lead to continuous families of holomorphic isometries into polydisks which are mutually distinct modulo automorphisms of the domain and target manifolds.

A holomorphic isometric embedding $f: \Delta \rightarrow \Omega$ is said to be asymptotically geodesic at $b \in \partial \mathcal{H}$ if and only if the length $\|\sigma\|$ of the second fundamental form $\sigma$ of the holomorphic isometry $f$ extends to a neighborhood of $b$ in $\bar{\Delta}$ as a continuous function vanishing on the boundary circle. In this article we study non-standard holomorphic isometries of the Poincaré disk into bounded symmetric domains which are asymptotically totally geodesic at a general point of the boundary circle. For the sake of simplicity we restrict ourselves to the case where the target domain $\Omega$ is an irreducible bounded symmetric domain, or more generally where $\Omega$ is a Cartesian product of identical irreducible bounded symmetric domains. Starting with the structural equation for holomorphic isometries arising from the Gauss equation, we obtain by covariant differentiation an identity relating certain holomorphic bisectional curvatures to the boundary behavior of the square of the norm of the second fundamental form of the holomorphic isometry. Using the nonpositivity of holomorphic bisectional curvatures on a bounded symmetric domain, for a non-standard holomorphic isometry $F: \Delta \rightarrow \Omega$ which is asymptotically totally geodesic at a general boundary point on the unit circle we prove that $\|\sigma\|$ must vanish at a general boundary point either to the order 1 or to the order $\frac{1}{2}$, giving a priori two types of possible asymptotic behavior of non-standard holomorphic isometric embeddings. When the vanishing order is precisely 1 resp. precisely $\frac{1}{2}$ for all general point of the boundary circle we will call such a map a non-standard holomorphic isometry of the Poincaré disk of the first resp. second kind. For the proof of the main result on the asymptotic 
behavior of the second fundamental form, starting from the structural equation arising from the Gauss equation the analysis is made possible by the existence of normal forms of $(1,0)$ tangent vectors. Geometrically, the embedded Poincaré disk is tangent to a moving family of totally geodesic polydisks, whose axes define a moving family of stretching and contracting principal directions. This geometric description allows us to study the asymptotic behavior of non-standard holomorphic embeddings of the Poincaré disk despite the in general very complicated boundary structure of bounded symmetric domains in their Harish-Chandra realizations (cf. Wolf $[\mathrm{Wo}]$ ).

In the second half of the article we deal with special cases of non-standard holomorphic isometric embeddings of the Poincaré disk, including especially those arising from Mok [Mk4]. We show first of all that any non-standard holomorphic isometric embedding of the Poincaré disk must be asymptotically totally geodesic at a general boundary point and in fact of the first kind whenever the target domain is a Cartesian product of complex unit balls. The proof exploits the constancy of holomorphic sectional curvatures, and the same argument fails in general for holomorphic isometric embeddings into arbitrary bounded symmetric domains. For the analysis of holomorphic isometries into the Siegel upper half-plane the asymptotic behavior is much more difficult due to the fact that the the isotropy subgroup does not act transitively on the space of unit $(1,0)$ tangent vectors at a given point. In the major part of the second half of the article we study the boundary behavior of the example of holomorphic isometric embedding from the Poincaré disk into a Siegel upper half-plane by an explicit determination of the boundary behavior of holomorphic sectional curvatures in the directions tangent to the embedded Poincaré disk. We show that this map is indeed asymptotically totally geodesic at a general point of the boundary circle and that moreover, somewhat unexpectedly, it is a non-standard holomorphic isometry of the first kind. For the metric computation we make use of formulas for symplectic geometry on Siegel upper half-planes as given in Siegel [Si].

In a forthcoming article we will show that in fact any holomorphic isometry $F: \Delta \rightarrow \Omega$ in the context as discussed in the above must necessarily be asymptotically totally geodesic at a general boundary point, so that in general a non-standard holomorphic isometric embedding into $\Omega$ must be either of the first kind or of the second kind. So far all examples are of the first kind, and it remains unknown whether a holomorphic isometry of the second kind actually exists. A related question is the existence problem of non-standard holomorphic isometries of the Poincaré disk into bounded symmetric domains which extend holomorphically to some neighborhood of the closed unit disk, i.e., those from which no singularities develop along the boundary circle. Towards the end of the article we will explore a possible relation between two existence problems.

Acknowledgement. The author is honored to dedicate this article to Professor Wenjun $\mathrm{Wu}$ on the joyous occasion of his ninetieth birthday. He also wishes to thank Sui Chung Ng for carefully reading a draft of the article, and in particular for making an observation which led the author to reformulate and streamline the proof of Proposition 2.

$\S 1$ Asymptotically totally geodesic holomorphic embeddings of the Poincaré disk 


\section{into bounded symmetric domains}

(1.1) Preliminaries and statement of results In an article on Arithmetic Geometry concerning the characterization of commutators of Hecke correspondences, Clozel and Ullmo [CU] reduced the problem to a question of characterization of germs of holomorphic isometries from a bounded domain $\Omega$ into a Cartesian product of identical copies of itself. Here a Hecke correspondence $T$ is defined on a finite-volume quotient $X$ of $\Omega$, and it is given by a certain 'multi-valued' automorphism, thus corresponding to a totally geodesic complex submanifold $S \subset X \times X$ of the "diagonal type". Let $p$ be the number of pre-image points over a general point $x_{0} \in X$ of the finite projection map $\pi_{1}: S \subset X \times X$ to the first direct factor and define $f:\left(X, x_{0}\right) \rightarrow\left(X, x_{0}\right) \times \cdots \times\left(X, x_{0}\right)$, where there are $p$ Cartesian factors, by sending $x \in X$ sufficiently close to $x_{0}$ to $\left(f_{1}(x), \cdots, f_{p}(x)\right)$, where $f_{i}, 1 \leq i \leq p$, denote the $p$ separate branches of $\pi_{1}^{-1}$. In [CU] the authors reformulate the original problem to asking, for an algebraic correspondence $S$ on $X$ commuting with $T$, whether the germ of holomorphic map $f$ is totally geodesic. They proved that $f$ is necessarily a holomorphic isometry up to a normalizing constant with respect to the Bergman metric, and the problem was then reduced to a problem completely differential-geometric in nature, viz., to the problem of characterizing germs of holomorphic isometries up to a normalizing constant with respect to the Bergman metric in the context as described. By lifting $f$ to universal covering domains, the problem is reduced to characterizing germs of holomorphic isometries up to normalizing constants of $(\Omega, 0)$ into a Cartesian product of identical copies of $(\Omega, 0)$ satisfying a certain additional non-degeneracy assumption, where $\Omega \Subset \mathbb{C}^{N}$ is the bounded symmetric domain in its HarishChandra realization. In $[\mathrm{CU}]$ the authors observed that in the case where $\Omega$ is irreducible and of rank $\geq 2$, any such a germ of map $f$ is necessarily totally geodesic as a consequence of the proof of Hermitian metric rigidity of Mok [Mk1,2]. In the case where $\Omega$ is the unit disk $\Delta$, under the assumption that the normalizing constant is an integer, they proved that the germ $\operatorname{Graph}(f)$ extends as an affine-algebraic variety, proved that $S \subset X \times X$ is indeed totally geodesic by exploiting the underlying equivariance of $S$ with respect to the fundamental group of $X$, but conjectured that total geodesy of $f$ should follow without any global considerations. In Mok [Mk3] we give a new proof of affine-algebraic extension of $\operatorname{Graph}(f)$ making it applicable also to the general rank- 1 case when $\Omega$ is the complex unit ball $B^{n}$ and solved the analogue of the conjecture in [CU, loc. cit.] for the complex unit ball $B^{n}, n \geq 2$. On the other hand, quite unexpectedly Mok [Mk4] found examples of holomorphic isometries of the Poincaré disk into the polydisk which are non-standard in the sense that they fail to be totally geodesic. To streamline the presentation in this article we will only consider the case where the bounded symmetric domain $\Omega$ is irreducible or where $\Omega$ is a Cartesian product of identical irreducible bounded symmetric domains. In Mok [Mk4] we study the general extension problem for germs of holomorphic isometries up to normalizing constants between bounded domains equipped with the Bergman metric. It was found that the issue of algebraic extension can be reduced to a question of rationality of the Bergman kernel. Since $\left(\Omega, d s_{\Omega}^{2}\right)$ is a complete Kähler manifold and since for a bounded symmetric domain $\Omega \Subset \mathbb{C}^{N}$ in its Harish-Chandra realization, the Bergman kernel $K_{\Omega}(z, w)$ is a rational function in $(z, \bar{w})$ on $\mathbb{C}^{N} \times \mathbb{C}^{N}(\mathrm{cf} . \mathrm{Xu}[\mathrm{Xu}])$, the results of Mok $[\mathrm{Mk} 4]$ in the special case of germs of holomorphic 
isometries of the Poincaré disk gives

Theorem (special case of Mok [Mk4]). Let $\left(\Delta, d s_{\Delta}^{2}\right)$ be the Poincaré disk of constant Gaussian curvature -1 . Let $\Omega \Subset \mathbb{C}^{N}$ be a bounded symmetric domain in its Harish-Chandra realization and denote by $g_{\Omega}$ the Bergman metric on $\Omega$. Let $\lambda>0$ be an arbitrary positive number and let $f:\left(\Delta, 0 ; \lambda d s_{\Delta}^{2}\right) \rightarrow\left(\Omega, 0 ; g_{\Omega}\right)$ be a germ of holomorphic isometry. Then $f$ extends to a proper holomorphic isometric embedding $F: \Delta \rightarrow \Omega$. Furthermore, the germ of subvariety $\operatorname{Graph}(f)$ at $(0,0)$ in $\Delta \times \Omega \Subset \mathbb{C} \times \mathbb{C}^{N}$ extends as an affine-algebraic variety $V \subset \mathbb{C} \times \mathbb{C}^{N}$. Moreover, $V \cap(\Delta \times \Omega)=\operatorname{Graph}(F)$.

We note here that $d s_{\Delta}^{2}$ is equivalently the Bergman metric on the unit disk $\Delta$. For the purpose of computation we will make use of the upper half-plane in place of the unit disk as the model of the Poincaré disk. Denote by $\mathcal{H}=\{\tau \in \mathbb{C}: \operatorname{Im} \tau>0\}, \tau=s+i t$, the upper half-plane. Recall that $\Omega \Subset \mathbb{C}^{N}$ is a bounded symmetric domain in its Harish-Chandra realization. We denote by $d s_{\mathcal{H}}^{2}$ the Poincaré metric of constant Gaussian curvature -1 on the upper half-plane $\mathcal{H}$. In the same article [Mk4] another example of a holomorphic isometry of the Poincaré disk into a Siegel upper half-plane was also constructed. When $\Omega$ is irreducible or when $\Omega$ is the Cartesian product of a number of identical irreducible bounded symmetric domains, which we assume throughout the rest of the current article, all Aut $(\Omega)$-invariant Kähler metrics are scalar multiples of each other. For convenience in curvature computations, in this article $d s_{\Omega}^{2}$ will stand for the choice of $\operatorname{Aut}(\Omega)$-invariant Kähler metric with respect to which all minimal disks on $\Omega$ are of constant Gaussian curvature -1 . Let $\lambda$ be some positive constant and $f:\left(\mathcal{H}, \lambda d s_{\mathcal{H}}^{2}\right) \rightarrow\left(\Omega, d s_{\Omega}^{2}\right)$ be a holomorphic isometry. At all but a finite number of boundary points $b \in \partial \mathcal{H}, f$ can be analytically continued to $F$ on a neighborhood $U_{b}$ of $b$ such that $F\left(U_{b} \cap \partial \mathcal{H}\right) \subset \partial \Omega$.

In this article we study the asymptotic behavior of holomorphic isometries of the Poincaré disk into bounded symmetric domains at a general point $b \in \partial \mathcal{H}$ of the real line. In preparation for such a study we show that the square of the norm of the second fundamental form extends as a real-analytic function to a neighborhood of a general boundary point $b \in \partial \mathcal{H}$. More precisely, we have

Proposition 1. Let $\Omega \Subset \mathbb{C}^{N}$ be a bounded symmetric domain in its Harish-Chandra realization which is assumed to be either irreducible or a product of identical irreducible bounded symmetric domains. Let $\lambda$ be a positive real number and let $F:\left(\mathcal{H}, \lambda d s_{\mathcal{H}}^{2}\right) \rightarrow\left(\Omega, d s_{\Omega}^{2}\right)$ be a holomorphic isometry. Let $b \in \partial \mathcal{H}$ be a boundary point and $U_{b}$ be an open neighborhood of $b$ in $\mathcal{H}$ such that $F$ can be analytically continued to $U_{b}$ and such that the extended map defines a holomorphic embedding of $U_{b}$ onto a locally complex submanifold $S_{b} \Subset \mathbb{C}^{N}$. Then, on $U_{b} \cap \mathcal{H}$ the function $\varphi=\|\sigma\|^{2}$ is a quotient $\frac{p(s, t)}{q(s, t)}$ where $p$ and $q$ are real-analytic functions in $s=\operatorname{Re}(\tau)$ and $t=\operatorname{Im}(\tau)$. Moreover, for all but a finite number of such points $b \in \partial \mathcal{H}$ and for $U_{b}$ sufficiently small, $\varphi$ extends to a real-analytic function on $U_{b}$.

Proof. On $S_{b} \subset \mathbb{C}^{N}$ write $Z=d F\left(\frac{\partial}{\partial \tau}\right)$, which is a holomorphic vector field on $S_{b}$. On $F\left(U_{b} \cap \mathcal{H}\right) \subset S_{b} \cap \Omega$ the second fundamental form $\sigma$ is obtained as follows. Let $x \in S_{b} \cap \Omega$. Extend $Z$ holomorphically to a holomorphic vector field, still to be denoted by the same symbol $Z$, on an open neighborhood $V$ of $x$ in $\Omega$. Denote by $\left\langle\cdot,,^{-}\right\rangle$the Hermitian inner product 
of the Kähler metric $d s_{\Omega}^{2}$, by $\|\cdot\|$ the associated norm, by $\left(g_{i \bar{j}}\right)$ the matrix expression of $d s_{\Omega}^{2}$ in terms of Euclidean coordinates on $\mathbb{C}^{N}$, and by $\left(g^{i \bar{j}}\right)$ the conjugate inverse of $\left(g_{i \bar{j}}\right)$. Write $\nabla$ for covariant differentiation on $\Omega$ with respect to $d s_{\Omega}^{2}$. Then, $\sigma_{x}: T_{x}\left(S_{b}\right) \times T_{x}\left(S_{b}\right) \rightarrow N_{S_{b} \mid \Omega, x}$ is given by $\sigma_{x}(Z, Z)=\operatorname{pr}^{\perp}\left(\nabla_{Z} Z\right)$, where $\mathrm{pr}^{\perp}$ signifies orthogonal projection from $T_{x} \Omega$ onto $N_{S_{b} \mid \Omega, x}$ with respect to $d s_{\Omega}^{2}$, given by $\operatorname{pr}^{\perp}(\eta)=\eta-\frac{\langle\eta, \overline{Z(x)}\rangle}{\|Z(x)\|^{2}} Z(x)$. Now covariant differentiation can be expressed in terms of Riemann-Christoffel symbols $\Gamma_{i j}^{k}$ with respect to the Euclidean coordinates $\left(z_{i}\right)$, given by

$$
\Gamma_{i j}^{k}=\sum_{\ell} g^{k \bar{\ell}} \frac{\partial g_{i \bar{\ell}}}{\partial z_{j}}, \quad \text { where } \quad g_{i \bar{\ell}}=A_{\Omega} \frac{\partial^{2}}{\partial z_{i} \partial \overline{z_{j}}} \log K_{\Omega}(z, z),
$$

where $K_{\Omega}$ is the Bergman kernel of $\Omega$, and $A_{\Omega}$ is some positive constant depending on $\Omega$. As is well-known, $K_{\Omega}(z, w)$ is a rational function in $(z, \bar{w})$ (cf. $\mathrm{Xu}[\mathrm{Xu}]$ ). On $U_{b} \subset \mathcal{H}$ we have $\varphi(\tau)=\left\|\sigma_{F(\tau)}\right\|^{2}$, where, writing $x=F(\tau) \in F\left(U_{b} \cap \mathcal{H}\right) \subset S_{b}$, we have $\left\|\sigma_{x}\right\|^{2}=\frac{\sigma_{x}(Z, Z)}{\|Z\|^{2}}$. From (1), the rationality of $K(z, w)$ in $(z, \bar{w})$, and the formula for the orthogonal projection $\operatorname{pr}^{\perp}: T_{x}(\Omega) \rightarrow N_{S_{b} \mid \Omega, x}$ it follows readily that $\varphi$ can be expressed as the quotient of two real-analytic functions over $U_{b}, \varphi=\frac{p(s, t)}{q(s, t)}$, as stated.

If $q(s, t)$ does not vanish on $U_{b} \cap \mathbb{R}$, then $\varphi$ already extends to $U_{b}$ as a real-analytic function. On the other hand, from the fact that $F:\left(\Delta, d s_{\Delta}^{2}\right) \rightarrow\left(\Omega, d s_{\Omega}^{2}\right)$ is a holomorphic isometry and from the Gauss equation (cf. the structural equation (1) in (1.2)) it follows readily that $\varphi$ is bounded on $U_{b} \cap \mathcal{H}$. Suppose now that $q(s, 0)$ vanishes for $s \in U_{b} \cap \mathbb{R}$. Choose $U_{b}$ to be a disk and write $q(s, t)=t^{e} u(s, t)$ on $U_{b}$, where $e>0$. From the boundedness of $\varphi$ on $U_{b} \cap \mathcal{H}$ it follows readily that $p(s, t)$ must vanish at any point $s \in U_{b} \cap \mathbb{R}$ at least to the order $e$. If $u(b, 0) \neq 0$, then shrinking $U_{b}$ if necessary $\varphi=\frac{p(s, t)}{q(s, t)}$ is indeed a real-analytic function on $U_{b}$.

In the argument above we have to avoid branch points of $F$ on $\partial \mathcal{H}$, points $b \in \partial \mathcal{H}$ where $F^{\prime}(b)=0$ and also points $b \in \partial \mathcal{H}$ where in the representation $\varphi=\frac{p(s, t)}{q(s, t)}$ the vanishing order of $q$ at $b$ exceeds the vanishing order $e$ of $q$ at a general point of $U_{b} \cap \partial \mathcal{H}$. We call any boundary point $b \in \mathbb{R}$ of the three types described a potentially bad boundary point, and we call a point $b \in \partial \mathcal{H}$ a bad boundary point if and only if either $b$ is a branch point of $F$ or $F$ is unbranched at $b$ but $\varphi$ fails to extend to a real-analytic function to some neighborhood $U_{b}$ of $b$ in $\mathbb{C}$. Otherwise $b \in \partial \mathcal{H}$ will be called a good boundary point. Obviously, all bad boundary points $b \in \partial \mathcal{H}$ are potentially bad boundary points, and to complete the proof of Proposition 1 it suffices to show that there are at most a finite number of potentially bad boundary points $b \in \mathbb{R}$.

To start with, note that in the argument concerning the real-analyticity of $\varphi$ on $U_{b}$ by means of an expression $\varphi=\frac{p(s, t)}{q(s, t)}$, the requirement that $F^{\prime}(b) \neq 0$ was imposed simply to make sure that $F\left(U_{b} \cap \mathcal{H}\right)=S_{b} \subset \mathbb{C}^{N}$ is a locally closed complex submanifold. Without that condition the whole argument still works since we can argue on $U_{b}$ in place of $S_{b}$ and consider covariant differentiation in terms of the pull-back of $\nabla$ to $U_{b} \cap \mathcal{H}$, i.e., we define the second fundamental form $\sigma$ of the subbundle $T_{U_{b}} \subset F^{*} T_{\mathbb{C}^{N}}$ over $U_{b} \cap \mathcal{H}$ and extend $\varphi=\|\sigma\|^{2}$ by means of the expression of $\varphi$ arising from Riemann-Christoffel symbols of the pulledback connection. ¿From preceding arguments we conclude that whenever $F$ is unbranched 
at $b \in \partial \mathcal{H}$, either $b$ is a good boundary point and every point $b^{\prime} \in \partial \mathcal{H}$ sufficiently close to $b$ is also a good boundary point, or $b$ is an isolated bad boundary point. This is so even if $F^{\prime}(b)=0$. Note that $b$ is an isolated bad boundary point only if the vanishing order of $q(s, t)$ at $b$ exceeds the generic vanishing order of $q(s, t)$ on $U_{b} \cap \partial \mathcal{H}$.

For a branch point $b \in \partial \mathcal{H}$ of $F$, the latter can be considered as a multivalent mapping on a neighborhood $U_{b}$ of $b$. More precisely, let $V \subset \mathbb{C} \times \mathbb{C}^{N}$ be an irreducible affine-algebraic curve which contains $\operatorname{Graph}(F)$ as an open subset. Note that $F$ extends continuously to $\partial \mathcal{H}=\mathbb{R}$ and it still makes sense to write $F(s)$ for $s \in \mathbb{R}$. Let $U_{b} \Subset \mathbb{C}$ be an open disk neighborhood of $b$ in $\mathbb{C}$ such that $b$ is the only branch point of $F$ on some neighborhood of $\overline{U_{b}}$, $B \Subset \mathbb{C}^{N}$ be an open neighborhood of $F(b)$ such that $F\left(U_{b} \cap \mathcal{H}\right) \subset B$. Let $E$ be the irreducible component of $\left(U_{b} \times B\right) \cap V$ which contains $\operatorname{Graph}\left(\left.F\right|_{U_{b} \cap \mathcal{H}}\right)$. Denote by $\rho: D \rightarrow E$ the normalization of $E$, noting that $D$ is nonsingular. Write $\operatorname{pr}_{1}: \mathbb{C} \times \mathbb{C}^{N} \rightarrow \mathbb{C}$ for the canonical projection onto the first direct factor, and $\mathrm{pr}_{2}: \mathbb{C} \times \mathbb{C}^{N} \rightarrow \mathbb{C}^{N}$ for the canonical projection onto the second direct factor. Define $G:=\operatorname{pr}_{2} \circ \rho$. Then, $G: D \rightarrow \mathbb{C}^{N}$ is a holomorphic map. $W:=\rho^{-1}\left(\operatorname{Graph}\left(\left.F\right|_{U_{b} \cap \mathcal{H}}\right)\right)$ is an open subset whose boundary $\partial W$ in $D$ is a closed semi-analytic subset of a real-analytic curve $\gamma$ defined by $\gamma=\left\{w \in D: \operatorname{Im}\left(\operatorname{pr}_{1}(\rho(w))=0\right\}\right.$, which is reducible. On $W \subset D$ define $\psi:=\varphi \circ \operatorname{pr}_{1} \circ \rho$. Using the same argument as in the last paragraph it remains possible to write $\psi=\frac{p}{q}$, where $p$ and $q$ are real-analytic functions on $D$. The branch point $b \in \partial \mathcal{H}$ corresponds to a point $\widetilde{b} \in \partial W \subset \gamma$ on the real-analytic curve and $G$ is unramified on $D$ except at $\widetilde{b}$. Let $b^{\prime} \in U_{b} \cap \partial \mathcal{H}$ be a boundary point other than $b$ and denote by $\widetilde{b^{\prime}} \in \partial W \subset \gamma$ the point corresponding to $b^{\prime}$. Then $\varphi$ is real-analytic at $b^{\prime}$ if and only if $\psi$ is real-analytic at $\widetilde{b^{\prime}} .\left(U_{b}-\{b\}\right) \cap \partial \mathcal{H}$ consists of two connected intervals $I_{1}$ and $I_{2}$. Each $I_{k} ; k=1,2$ corresponds to an open subset $J_{k}$ of an irreducible component $\gamma_{k}$ of $\gamma$. Fix $k=1$ or 2 and denote by $e_{k}$ the generic vanishing order of $q$ at a smooth point of $\gamma_{k}$. If $e_{k}=0$ then $q$ is non-zero on $\gamma_{k}$ outside a finite number of points. In particular, there can only be a finite number of potentially bad boundary points on $I_{k}$. On the other hand, if $e_{k}>0$ then for all but a finite number of points on $\gamma_{k}$, the curve $\gamma_{k}$ is smooth at the point and the vanishing order of $q$ at the point is exactly equal to $e_{k}$. The argument using the boundedness of $\varphi$ previously then applies verbatim on $\psi$ to show that there are at most a finite number of potentially bad boundary points on $I_{k}$. Applying the argument to both $\gamma_{1}$ and $\gamma_{2}$ we conclude therefore that there are only a finite number of potentially bad boundary points on $U_{b} \cap \partial \mathcal{H}$. We conclude therefore that $\varphi$ is real-analytic at every boundary point $b \in \partial \mathcal{H}$ with the exception of an at worst discrete set of bad boundary points $b \in \partial \mathcal{H}$. Finally, by means of a fractional linear transformation $\Phi \in \operatorname{SL}(2, \mathbb{R})$ the infinity point $\infty$ can be transformed to a finite point $a$ in $\mathbb{R}$, and a sufficiently large real point $s \in \mathbb{R}$ is transformed to a point $\Phi(s)$ close to $a$. Thus any sufficiently large real point $s$ is not a bad boundary point. As a consequence, there are only a finite number of potentially bad boundary points on $\partial \mathcal{H}$, hence only a finite number of bad boundary points, and the proof of Proposition 1 is complete.

The main result of the article concerns asymptotic behavior of holomorphic isometries of the Poincaré disk into bounded symmetric domains, as follows. 
Theorem 1. Let $\lambda$ be a positive real number and let $f:\left(\Delta, \lambda d s_{\Delta}^{2}\right) \rightarrow\left(\Omega, d s_{\Omega}^{2}\right)$ be a holomorphic isometry of the Poincaré disk into a bounded symmetric domain $\Omega$ which is either irreducible or a Cartesian product of identical irreducible bounded symmetric domains. Equip $\Omega$ with an Aut $(\Omega)$-invariant complete Kähler metric $d s_{\Omega}^{2}$ such that the minimal disks are of constant holomorphic sectional curvature -1 . Suppose $f$ is not totally geodesic and it is asymptotically geodesic at a general boundary point. Then, the length of the second fundamental form $\|\sigma\|$ must vanish to the order 1 or $\frac{1}{2}$ at a general boundary point $b$ of $\Delta$, i.e., at a general point of the unit circle $S^{1}=\partial \Delta$. In other words, the real-analytic function $\varphi$ defined on a neighborhood of $b$ must vanish either to the order 2 resp. 1.

As the starting point of the proof of Theorem 1, by making use of the structural equation which arises from the definition of holomorphic isometries, we will obtain a curvature formula for bisectional curvatures which involve the second fundamental form of the holomorphic isometry $f: \Delta \rightarrow \Omega$.

(1.2) Structural equation for holomorphic isometries Since $f:\left(\mathcal{H}, \lambda d s_{\mathcal{H}}^{2}\right) \rightarrow\left(\Omega, d s_{\Omega}^{2}\right)$ is by assumption totally geodesic at a general boundary point of $\partial \mathcal{H}$, the isometric constant $\lambda$ must agree with those of totally geodesic Poincaré disks $D$ on $\Omega$. By the choice of normalization of $d s_{\Omega}^{2}$, we must have $\lambda=k$, where $1 \leq k \leq r:=\operatorname{rank}(\Omega)$ is a positive integer, in which case $D$ is of constant Gaussian curvature $-\frac{1}{k}$. The latter holds true if and only if there exists a totally geodesic polydisk $P \subset \Omega$ of complex dimension $k$, and there is a biholomorphism $\gamma \in \operatorname{Aut}(\Omega)$ such that $\gamma(D)$ is the diagonal of $P$. For the holomorphic isometry $f:\left(\mathcal{H}, k \cdot d s_{\mathcal{H}}^{2}\right) \rightarrow\left(\Omega, d s_{\Omega}^{2}\right)$, denote the image $f(\mathcal{H})$ by $S$, which is an embedded holomorphic curve on $\Omega$. Write $T_{S}$ resp. $T_{\Omega}$ for the holomorphic tangent bundle of $S$ resp. $\Omega$ and identify the holomorphic tangent bundle with the $(1,0)$ component of the complexified tangent bundle. Denote by $R$ the curvature tensor of $\left(\Omega, d s_{\Omega}^{2}\right)$ and by $\sigma: T_{S} \times T_{S} \rightarrow N_{S \mid \Omega}$ the second fundamental form (restricted to $(1,0)$ tangent vectors), where $N_{S \mid \Omega}$ denotes the holomorphic normal bundle of $S$ in $\Omega$, which is identified in what follows with the orthogonal complement of $T_{S}$ in $T_{\Omega}$ as a smooth bundle. By the Gauss equation, for any $x \in S, \alpha \in T_{x}(X)$, we have the structural equation

$$
\begin{gathered}
R_{\alpha \bar{\alpha} \alpha \bar{\alpha}}-\|\sigma\|^{2}\|\alpha\|^{4}=-\frac{1}{k}\|\alpha\|^{4} \\
R_{\alpha \bar{\alpha} \alpha \bar{\alpha}}=\left(-\frac{1}{k}+\varphi\right)\langle\alpha, \bar{\alpha}\rangle^{2}
\end{gathered}
$$

where $\|\cdot\|$ denotes the norm on $\left(\Omega, d s_{\Omega}^{2}\right)$ and $\left\langle\cdot,{ }^{-}\right\rangle$denotes the corresponding Hermitian inner product. Since $\left(\Omega, d s_{\Omega}^{2}\right)$ is locally symmetric, the curvature tensor $R$ is parallel, i.e. $\nabla R=0$. In what follows $\alpha$ will denote instead a holomorphic vector field tangent to $S$ defined on some open neighborhood of $x \in S$. Further specification on $\alpha$ will be made later on in the calculation. Differentiating (1) with respect to $\tau$ we have

$$
\begin{gathered}
2 R\left(\nabla_{f_{*}\left(\frac{\partial}{\partial \tau}\right)} \alpha, \bar{\alpha} ; \alpha, \bar{\alpha}\right) \\
=\frac{\partial \varphi}{\partial \tau}\|\alpha\|^{4}+2\left(-\frac{1}{k}+\varphi\right)\left\langle\nabla_{f_{*}\left(\frac{\partial}{\partial \tau}\right)} \alpha, \bar{\alpha}\right\rangle\|\alpha\|^{2} .
\end{gathered}
$$


Differentiating (2) with respect to $\bar{\tau}$ we have

$$
\begin{gathered}
4 R\left(\nabla_{f_{*}\left(\frac{\partial}{\partial \tau}\right)} \alpha, \overline{\nabla_{f_{*}\left(\frac{\partial}{\partial \tau}\right)}} ; \alpha, \bar{\alpha}\right)+2 R\left(\nabla \frac{\partial_{f_{*}\left(\frac{\partial}{\partial \tau}\right)}}{f_{*}\left(\frac{\partial}{\partial \tau}\right)} \alpha, \bar{\alpha} ; \alpha, \bar{\alpha}\right) \\
=\frac{\partial^{2} \varphi}{\partial \tau \partial \bar{\tau}}\|\alpha\|^{4}+2 \frac{\partial \varphi}{\partial \tau}\left\langle\alpha, \overline{\nabla_{f_{*}\left(\frac{\partial}{\partial \tau}\right)} \alpha}\right\rangle\|\alpha\|^{2}+2 \frac{\partial \varphi}{\partial \bar{\tau}}\left\langle\nabla_{f_{*}\left(\frac{\partial}{\partial \tau}\right)} \alpha ; \bar{\alpha}\right\rangle\|\alpha\|^{2} \\
+2\left(-\frac{1}{k}+\varphi\right)\left\langle\nabla \frac{1}{f_{*\left(\frac{\partial}{\partial \tau}\right)}} \nabla_{f_{*}\left(\frac{\partial}{\partial \tau}\right)} \alpha, \bar{\alpha}\right\rangle\|\alpha\|^{2}+2\left(-\frac{1}{k}+\varphi\right)\left\langle\nabla_{f_{*}\left(\frac{\partial}{\partial \tau}\right)} \alpha, \overline{\nabla_{f_{*}\left(\frac{\partial}{\partial \tau}\right)} \alpha}\right\rangle\|\alpha\|^{2} \\
+2\left(-\frac{1}{k}+\varphi\right)\left\langle\nabla_{f_{*}\left(\frac{\partial}{\partial \tau}\right)} \alpha, \bar{\alpha}\right\rangle\left\langle\alpha, \overline{\nabla_{f_{*}\left(\frac{\partial}{\partial \tau}\right)} \alpha}\right\rangle .
\end{gathered}
$$

Assume now $x \in \mathcal{H} \cap U_{b}$. Choose the holomorphic vector field $\alpha$ on a neighborhood of $f(x)$ to be the coordinate vector field $\frac{\partial}{\partial \zeta}$ with respect to a complex geodesic coordinate $\zeta$ at $x$, so that $\|\alpha(x)\|=1$ and $\nabla_{\alpha}^{S} \alpha(x)=0$ for the connection $\nabla^{S}$ on $S$ induced from the connection $\nabla$ on $\left(\Omega, d s_{\Omega}^{2}\right)$. Thus, $\nabla_{\alpha} \alpha(x)$ is orthogonal to $\alpha(x)$, so that $\nabla_{\alpha} \alpha(x)=\sigma(\alpha, \alpha)(x)$ for the second fundamental form $\sigma$ of $S$ in $\Omega$. Write $\mu(x):=\sigma(\alpha, \alpha)(x)$. Evaluating at $x$ we have

$$
\begin{gathered}
4 R\left(\nabla_{f_{*}\left(\frac{\partial}{\partial \tau}\right)} \alpha, \overline{\nabla_{f_{*}\left(\frac{\partial}{\partial \tau}\right)} \alpha} ; \alpha, \bar{\alpha}\right)(x)-2 R\left(R_{f_{*}\left(\frac{\partial}{\partial \tau}\right) \overline{f_{*}\left(\frac{\partial}{\partial \tau}\right)}}, \bar{\alpha} ; \alpha, \bar{\alpha}\right)(x) \\
=\frac{\partial^{2} \varphi}{\partial \tau \partial \bar{\tau}}(x)+2\left(-\frac{1}{k}+\varphi(x)\right)\left\langle-R_{f_{*}\left(\frac{\partial}{\partial \tau}\right) \overline{f_{*}\left(\frac{\partial}{\partial \tau}\right)} \alpha}, \bar{\alpha}\right\rangle(x)+2\left(-\frac{1}{k}+\varphi(x)\right)\left\|\nabla_{f_{*}\left(\frac{\partial}{\partial \tau}\right)} \alpha(x)\right\|^{2} .
\end{gathered}
$$

Here, writing $f_{*}\left(\frac{\partial}{\partial \tau}\right)=\gamma \alpha$, we have $\nabla_{f_{*}\left(\frac{\partial}{\partial \tau}\right)} \alpha(x)=\gamma(x) \nabla_{\alpha} \alpha(x)=\gamma(x) \mu(x)$, which is orthogonal to $\alpha(x) \in T_{x}(S)$. The Poincaré metric of constant Gaussian curvature $-\frac{1}{k}$ on $\mathcal{H}$ is given by $2 \operatorname{Re}\left(\frac{k d \tau \otimes d \bar{\tau}}{2 t^{2}}\right)$, so that $\left\|f_{*}\left(\frac{\partial}{\partial \tau}\right)\right\|=\sqrt{\frac{2}{k}}\left(\frac{1}{t}\right)$. Here the constants can be checked by verifying that $2 \operatorname{Re}\left(\frac{d \tau \otimes d \bar{\tau}}{2 t^{2}}\right)$ corresponds via the Cayley transform to the Hermitian metric $2 \operatorname{Re}\left(\frac{2 d z \otimes d \bar{z}}{\left(1-|z|^{2}\right)^{2}}\right)$ on the unit disk, which is of constant Gaussian curvature -1 . ¿From (4) we derive

$$
\begin{gathered}
\frac{2 k}{t^{2}} R_{\mu \bar{\mu} \alpha \bar{\alpha}}(x)-\frac{\partial^{2} \varphi}{\partial \tau \partial \bar{\tau}}(x) \\
=2\left\|f_{*}\left(\frac{\partial}{\partial \tau}\right)\right\|^{2} R\left(R_{\alpha \bar{\alpha} \alpha}, \bar{\alpha} ; \alpha, \bar{\alpha}\right)+2\left(-\frac{1}{k}+\varphi(x)\right)\left(-\left\|f_{*}\left(\frac{\partial}{\partial \tau}\right)\right\|^{2} R_{\alpha \bar{\alpha} \alpha \bar{\alpha}}+\left\|f_{*}\left(\frac{\partial}{\partial \tau}\right)\right\|^{2}\|\mu(x)\|^{2}\right) \\
=2\left\|f_{*}\left(\frac{\partial}{\partial \tau}\right)\right\|^{2}\left(R\left(R_{\alpha \bar{\alpha} \alpha}, \bar{\alpha} ; \alpha, \bar{\alpha}\right)+\left(-\frac{1}{k}+\varphi(x)\right)\left(-R_{\alpha \bar{\alpha} \alpha \bar{\alpha}+\varphi(x))}\right.\right. \\
=\frac{k}{t^{2}}\left(R\left(R_{\alpha \bar{\alpha} \alpha}, \bar{\alpha} ; \alpha, \bar{\alpha}\right)+\left(-\frac{1}{k}+\varphi(x)\right)\left(\frac{1}{k}\right)\right)
\end{gathered}
$$

which will be the basis of further calculations for the proof of Theorem 1 in the next subsections.

(1.3) Proof of Theorem 1 when the target bounded symmetric domain is of rank 2 Consider the special case where $r=2$ and $k=1$. Writing $\alpha=\alpha_{1} e_{1}+\alpha_{2} e_{2}$ in normal form, we have

$$
-R_{\alpha \bar{\alpha} \alpha \bar{\alpha}}=\alpha_{1}^{4}+\alpha_{2}^{4}=\left(\alpha_{1}^{2}+\alpha_{2}^{2}\right)^{2}-2 \alpha_{1}^{2} \alpha_{2}^{2}=1-2 \alpha_{1}^{2} \alpha_{2}^{2}
$$


so that

$$
\varphi=\|\sigma\|^{2}=2 \alpha_{1}^{2} \alpha_{2}^{2} \quad \text { at } \quad x
$$

At the same time,

$$
R\left(R_{\alpha \bar{\alpha} \alpha}, \bar{\alpha} ; \alpha, \bar{\alpha}\right)=\alpha_{1}^{6}+\alpha_{2}^{6}=\left(\alpha_{1}^{2}+\alpha_{1}^{2}\right)^{3}-3 \alpha_{1}^{4} \alpha_{2}^{2}-2 \alpha_{1}^{2} \alpha_{2}^{4}=1-3 \alpha_{1}^{2} \alpha_{2}^{2}\left(\alpha_{1}^{2}+\alpha_{2}^{2}\right)=1-3 \alpha_{1}^{2} \alpha_{2}^{2} .
$$

¿From (5) for the special case of $r=2, k=1$ we conclude that

$$
\frac{2}{t^{2}} R_{\mu \bar{\mu} \alpha \bar{\alpha}}-\frac{\partial^{2} \varphi}{\partial \tau \partial \bar{\tau}}=\frac{1}{t^{2}}\left(1-3 \alpha_{1}^{2} \alpha_{2}^{2}-1+2 \alpha_{1}^{2} \alpha_{2}^{2}\right)=-\frac{1}{t^{2}}\left(\alpha_{1}^{2} \alpha_{2}^{2}\right)=-\frac{\varphi}{2 t^{2}} .
$$

By assumption $\varphi$ vanishes to the order $q \geq 1$ at every point of $I=\partial \mathcal{H} \cap U_{b}$. Writing $\varphi=t^{q} u$, where $u>0$ on $I$, we have

$$
\begin{gathered}
\frac{\partial^{2} \varphi}{\partial \tau \partial \bar{\tau}}=\frac{1}{4}\left(\frac{\partial^{2}}{\partial t^{2}}+\frac{\partial^{2}}{\partial s^{2}}\right)\left(t^{q} u\right) \\
=\frac{1}{4}\left((q(q-1)) t^{q-2} u+2 q t^{q-1} \frac{\partial u}{\partial t}+t^{q} \frac{\partial^{2} u}{\partial t^{2}}+t^{q} \frac{\partial^{2} u}{\partial s^{2}}\right) .
\end{gathered}
$$

On the other hand,

$$
\frac{\varphi}{2 t^{2}}=t^{q-2}\left(\frac{u}{2}\right)
$$

Hence,

$$
R_{\mu \bar{\mu} \alpha \bar{\alpha}}=\frac{t^{2}}{2}\left(\frac{\partial^{2} \varphi}{\partial \tau \partial \bar{\tau}}-\frac{\varphi}{2 t^{2}}\right)=\frac{1}{8}(q(q-1)-2) t^{q} u+\frac{q t^{q+1}}{4} \frac{\partial u}{\partial t}+\frac{t^{q+2}}{8}\left(\frac{\partial^{2} u}{\partial t^{2}}+\frac{\partial^{2} u}{\partial s^{2}}\right) .
$$

Since $\left(\Omega, d s_{\Omega}^{2}\right)$ is of nonpositive holomorphic bisectional curvature, the left-hand side is nonpositive. On the other hand, for $q \geq 3$ the right-hand side is positive for $t>0$ sufficiently small, yielding a contradiction. This proves Theorem 1 in the special case where $\Omega$ is of rank 2 and $k=1$. We have actually settled the case for $r=2$. In fact, by the Ahtfors-Schwarz Lemma, for any holomorphic map $h: \Delta \rightarrow \Omega$ we must have $h^{*} d s_{\Omega}^{2} \leq r d s_{\Delta}^{2}$. When $k=r$ and $F:\left(\Delta, r \cdot d s_{\Delta}^{2}\right) \rightarrow\left(\Omega, d s_{\Omega}^{2}\right)$ we have the extremal case $F^{*} d s_{\Omega}^{2}=r d s_{\Delta}^{2}$, and in this case $F$ must be a totally geodesic embedding. In particular, for $r=2, k=1$ is the only case for which Theorem 1 is relevant, which has been settled in the above.

(1.4) Proof of Theorem 1 in the general case

At $\tau \in \mathcal{H}$ for a unit vector $\alpha$ tangent to $S$ at $f(\tau)$ we write

$$
\alpha=\frac{1}{\sqrt{k}} \sum_{i=1}^{n} \beta_{i} e_{i}, \quad \beta_{1} \geq \beta_{2} \geq \cdots \geq \beta_{n} \geq 0,
$$

where $\beta_{i}=1+\nu_{i}$ for $1 \leq i \leq k$ and $\beta_{j}=\delta_{j-k}$ for $k+1 \leq j \leq n$. We call $\left\{e_{i}\right\}_{i=1}^{n}$ a set of principal directions. For $1 \leq i \leq k, \lim _{\tau \rightarrow p} \beta_{i}=1$, and we call $e_{i}, 1 \leq i \leq k$, a stretching principal direction. For $k+1 \leq j \leq n, \lim _{\tau \rightarrow p} \delta_{j}=0$ and we call $e_{j}$ a contracting principal 
direction. Note that the choice of $\left(e_{i}\right)_{i=1}^{n}$ may not be unique up to scalar multiplication by $e^{i \theta}$ but this does not affect the ensuing discussion. We proceed now to consider the stretching principal directions $e_{1}, \ldots, e_{k}$. ¿From $\|\alpha\|^{2}=1$ we have the identity

$$
\gamma_{1}+\cdots+\gamma_{k}=-\frac{1}{2}\left(\left(\gamma_{1}^{2}+\cdots+\gamma_{k}^{2}\right)+\left(\delta_{1}^{2}+\cdots+\delta_{r-k}^{2}\right)\right) .
$$

On the other hand,

$$
-k^{2} R_{\alpha \bar{\alpha} \alpha \bar{\alpha}}=\left(\left(1+\gamma_{1}\right)^{4}+\cdots+\left(1+\gamma_{k}\right)^{4}\right)+\left(\delta_{1}^{4}+\cdots \delta_{r-k}^{4}\right)
$$

so that by the structural equation (1) and by (14) we have

$$
\begin{gathered}
k^{2} \varphi=k+k^{2} R_{\alpha \bar{\alpha} \alpha \bar{\alpha}} \\
=-4\left(\gamma_{1}+\cdots+\gamma_{k}\right)-6\left(\gamma_{1}^{2}+\cdots+\gamma_{k}^{2}\right)-4\left(\gamma_{1}^{3}+\cdots+\gamma_{k}^{3}\right)-\left(\gamma_{1}^{4}+\cdots+\gamma_{k}^{4}\right)-\left(\delta_{1}^{4}+\cdots+\delta_{r-k}^{4}\right) \\
=\left(2\left(\gamma_{1}^{2}+\cdots+\gamma_{k}^{2}\right)+2\left(\delta_{1}^{2}+\cdots+\delta_{r-k}^{2}\right)\right)-6\left(\gamma_{1}^{2}+\cdots+\gamma_{k}^{2}\right)-4\left(\gamma_{1}^{3}+\cdots+\gamma_{k}^{3}\right) \\
-\left(\gamma_{1}^{4}+\cdots+\gamma_{k}^{4}\right)-\left(\delta_{1}^{4}+\cdots+\delta_{r-k}^{4}\right) \\
=-4\left(\gamma_{1}^{2}+\cdots+\gamma_{k}^{2}\right)+2\left(\delta_{1}^{2}+\cdots+\delta_{r-k}^{2}\right)-\left(\delta_{1}^{4}+\cdots+\delta_{r-k}^{4}\right)+E^{\prime}
\end{gathered}
$$

where $E^{\prime}$ is an error term which is a linear combination of sums of powers of $\gamma_{i}^{p}, 1 \leq i \leq k$, where $p \geq 3$. Furthermore,

$$
\begin{gathered}
k^{3} R\left(R_{\alpha \bar{\alpha} \alpha}, \bar{\alpha} ; \alpha, \bar{\alpha}\right)=\beta_{1}^{6}+\cdots+\beta_{r}^{6} \\
=k+6\left(\gamma_{1}+\cdots+\gamma_{k}\right)+15\left(\gamma_{1}^{2}+\cdots+\gamma_{k}^{2}\right)+20\left(\gamma_{1}^{3}+\cdots+\gamma_{k}^{3}\right)+15\left(\gamma_{1}^{4}+\cdots+\gamma_{k}^{4}\right) \\
+6\left(\gamma_{1}^{5}+\cdots+\gamma_{k}^{5}\right)+\left(\gamma_{1}^{6}+\cdots+\gamma_{k}^{6}\right)+\left(\delta_{1}^{6}+\cdots+\delta_{r-k}^{6}\right) \\
=k-3\left(\left(\gamma_{1}^{2}+\cdots+\gamma_{k}^{2}\right)+\left(\delta_{1}^{2}+\cdots+\delta_{r-k}^{2}\right)\right)+15\left(\gamma_{1}^{2}+\cdots+\gamma_{k}^{2}\right)+E^{\prime \prime} \\
=k+\left(6\left(\gamma_{1}^{2}+\cdots+\gamma_{k}^{2}\right)-3\left(\delta_{1}^{2}+\cdots+\delta_{r-k}^{2}\right)\right)+6\left(\gamma_{1}^{2}+\cdots+\gamma_{k}^{2}\right)-\frac{3}{2}+E^{\prime \prime} \\
=k-\frac{3 k^{2} \varphi}{2}+6\left(\gamma_{1}^{2}+\cdots+\gamma_{k}^{2}\right)-\frac{3}{2}\left(\delta_{1}^{4}+\cdots+\delta_{r-k}^{4}\right)+E_{0},
\end{gathered}
$$

where the error term $E^{\prime \prime}$ is a linear combination of sums of powers of $\gamma_{i}^{p}, 1 \leq i \leq k, p \geq 3$ and of $\delta_{\ell}^{6}, 1 \leq \ell \leq r-k$. The same description applies to $E_{0}$, which is a linear combination of $E^{\prime}$ and $E^{\prime \prime}$. Here in (17) the second equality is derived from (14), and the last line is derived from (16). The identity (5) then translates into

$$
\begin{gathered}
\frac{2 k}{t^{2}} R_{\mu \bar{\mu} \alpha \bar{\alpha}}-\frac{\partial^{2} \varphi}{\partial \tau \partial \bar{\tau}} \\
=\frac{k}{t^{2}}\left(\frac{1}{k^{2}}-\frac{3 \varphi}{2 k}+\frac{6}{k^{3}}\left(\gamma_{1}^{2}+\cdots+\gamma_{k}^{2}\right)-\frac{3}{2 k^{3}}\left(\delta_{1}^{4}+\cdots+\delta_{r-k}^{4}\right)+\frac{E_{0}}{k^{3}}+\left(-\frac{1}{k}+\varphi\right)\left(\frac{1}{k}\right)\right) \\
\left.=\frac{1}{t^{2}}\left(-\frac{\varphi}{2}+\frac{6}{k^{2}}\left(\gamma_{1}^{2}+\cdots+\gamma_{k}^{2}\right)\right)-\frac{3}{2 k^{2}}\left(\delta_{1}^{4}+\cdots+\delta_{r-k}^{4}\right)\right)+\frac{E_{0}}{k^{2} t^{2}},
\end{gathered}
$$

Writing $\varphi=t^{q} u$ as before we have

$$
\frac{2 k}{t^{2}} R_{\mu \bar{\mu} \alpha \bar{\alpha}}=\frac{\partial^{2} \varphi}{\partial \tau \partial \bar{\tau}}-\frac{\varphi}{2 t^{2}}+\frac{6}{k^{2} t^{2}}\left(\gamma_{1}^{2}+\cdots+\gamma_{k}^{2}\right)-\frac{3}{2 k^{2} t^{2}}\left(\delta_{1}^{4}+\cdots+\delta_{r-k}^{4}\right)+\frac{E_{0}}{k^{2} t^{2}},
$$


giving

$$
\begin{gathered}
R_{\mu \bar{\mu} \alpha \bar{\alpha}}=\frac{1}{8 k}(q(q-1)-2) t^{q} u+\frac{q t^{q+1}}{4 k} \frac{\partial u}{\partial t}+\frac{t^{q+2}}{8 k}\left(\frac{\partial^{2} u}{\partial t^{2}}+\frac{\partial^{2} u}{\partial s^{2}}\right) \\
+\frac{3}{k^{3}}\left(\gamma_{1}^{2}+\cdots+\gamma_{k}^{2}\right)-\frac{3}{4 k^{3}}\left(\delta_{1}^{4}+\cdots+\delta_{r-k}^{4}\right)+E .
\end{gathered}
$$

where $E=\frac{E_{0}}{2 k^{3}}$. The curvature expansion (20) is the analogue of (12) in the general case where the target domain $\Omega$ is of arbitrary rank $r \geq 2$ and where $f(\mathcal{H}) \subset \Omega$ is of constant Gaussian curvature $-\frac{1}{k}, 1 \leq k \leq r$. In the case where $k=1$ and $r=2$ the only $\gamma_{i}$ 's and $\delta_{j}$ 's are $\gamma=\gamma_{1}, \delta=\delta_{1}$, and we have from (15) $\delta^{2}=-2 \gamma-\gamma^{2}$. ¿From this it follows that $3 \gamma^{2}-\frac{3}{4} \delta^{4}=3 \gamma^{2}-\frac{3}{4}\left(2 \gamma+\gamma^{2}\right)^{2}=-3 \gamma^{3}-\frac{3}{4} \gamma^{4}$, which gives $-E$, the error term in $E$ generally of higher order in $\gamma_{i}$ 's and $\delta_{j}$ 's.

For the proof of Theorem 1 it remains to justify that the identity (20) would lead to a contradiction if $q \geq 3$. In the case where $r=2$ and $k=1$ contradiction was derived from the nonpositivity of the bisectional curvature $R_{\mu \bar{\mu} \alpha \bar{\alpha}}$ on the left-hand side of (20) and the positivity of the coefficient of the dominant term $t^{q}$ on the right-hand side of (20) when the latter is regarded as an asymptotic expansion in $t$ as $t=\operatorname{Re}(\tau)$ approaches to 0 . This special case suggests that the negative term involving $\delta_{j}^{4}$ 's on the right-hand cannot be ignored. In that case there is only one $\delta=\delta_{1}$, which is of the order of $\sqrt{|\gamma|}$, where $\gamma=\gamma_{1}$, and the expression $3 \gamma^{2}-\frac{3}{4} \delta^{4}$ consisting of two competing terms reduces to a term of higher vanishing order in $t$, i.e., the two competing terms in $3 \gamma^{2}-\frac{3}{4} \delta^{4}$ cancel each other up to terms of higher order.

We are going to derive a contradiction by studying the interplay among the terms $\varphi=$ $t^{q} u, \gamma_{1}^{2}+\cdots+\gamma_{k}^{2}$, both of which are attached to positive coefficients, and the term $\delta_{1}^{4}+\cdots+\delta_{r-k}^{4}$, which is attached to a negative coefficient. For the argument we introduce some notations, as follows. For each integer $\ell \geq 1$ we denote by $\Gamma_{\ell}:=\left|\gamma_{1}\right|^{\ell}+\cdots+\left|\gamma_{k}\right|^{\ell}, \Delta_{\ell}:=\left|\delta_{1}^{\ell}\right|+\cdots+\left|\delta_{r-k}\right|^{4}$. Recall that a boundary point $b \in \partial \mathcal{H}$ has been chosen where the function $\varphi=t^{q} u$ is smooth and where $u(b) \neq 0$. To derive a contradiction to $q \geq 3$ it suffices to show that for a general point $b \in \partial \mathcal{H}$, the right-hand side of (20) becomes strictly positive for $\tau \in \mathcal{H}$ sufficiently close to $b$. Recall that $U$ is a sufficiently small neighborhood of $b$ in $\mathbb{C}$. For a positive function $h$ on $U \cap \mathcal{H}$, the expression $O(h)$ means a function $s$ such that $|s| \leq K h$ for some positive constant $K$, the expression $o(h)$ means a function $s$ such that $|s| \leq L(\tau) h$ for a positive function $L(\tau)$ on $U \cap \mathcal{H}$ such that $L(\tau)$ tends to 0 as $\tau$ tends to $b$. With these conventions the identity (20) can be rewritten as

$$
R_{\mu \bar{\mu} \alpha \bar{\alpha}}=\frac{1}{8 k}(q(q-1)-2) \varphi+o(\varphi)+\frac{3}{k^{3}} \Gamma_{2}-\frac{3}{4 k^{3}} \Delta^{4}+O\left(\Gamma_{3}\right)+O\left(\Delta_{6}\right) .
$$

The precise coefficients attached to $\varphi, \Gamma_{2}$ and $\Delta_{4}$ will not matter; only the signs of the coefficients matter. To derive a contradiction it suffices to prove

Lemma 1. Let $A, B$ and $C$ be positive real numbers. Then, for any point $\tau \in U \cap \mathcal{H}$ sufficiently close to $b$ we have

$$
\begin{gathered}
A \varphi(\tau)+B \Gamma_{2}(\tau)-C \Delta_{4}(\tau)+o(\varphi)(\tau)+O\left(\Gamma_{3}\right)(\tau)+O\left(\Delta_{6}\right)(\tau)>0 \\
12
\end{gathered}
$$


Proof. Clearly $\left(\max \left|\gamma_{i}\right|\right)^{\ell} \leq \Gamma_{\ell} \leq k\left(\max \left|\gamma_{i}\right|\right)^{\ell},\left(\max \left|\delta_{j}\right|\right)^{\ell} \leq \Delta_{\ell} \leq(n-k)\left(\max \left|\delta_{j}\right|\right)^{\ell}$. As a consequence, we have $O\left(\Gamma_{3}\right)=o\left(\Gamma_{2}\right)$ and $O\left(\Delta_{6}\right)=o\left(\Delta_{4}\right)$. By choosing $\epsilon>0$ sufficiently small so that $A^{\prime}:=A-\epsilon>0, B^{\prime}=B-\epsilon>0$, and $C^{\prime}:=C-\epsilon>0$, to prove Lemma 1 it suffices to show that $A^{\prime} \varphi(\tau)+B^{\prime} \Gamma_{2}(\tau)-C \Delta_{4}(\tau)>0$ for $\tau \in U \cap \mathcal{H}$ sufficiently closed to a given general point $b \in \partial \mathcal{H}$. Since Lemma 1 is to be established for any positive real numbers $A, B$ and $C$ without loss of generality we can drop the error terms in the statement of Lemma 1 and just proceed to show that $A \varphi(\tau)+B \Gamma_{2}(\tau)-C \Delta_{4}(\tau)>0$ for $\tau$ sufficiently close to $b$. There are positive absolute constants $a$ and $b$ such that $a\left(\Delta_{2}\right)^{2}<\Delta_{4}<b\left(\Delta_{2}\right)^{2}$. On the other hand, in view of (16) the three nonnegative functions $\varphi, \Gamma_{2}$ and $\Delta_{2}$ are related by

$$
\begin{gathered}
\Delta_{2}=\frac{k^{2}}{2} \varphi+2 \Gamma_{2}+o\left(\Gamma_{2}\right)+o\left(\Delta_{2}\right) ; \text { so that } \\
\Delta_{2}=O(\varphi)+O\left(\Gamma_{2}\right) .
\end{gathered}
$$

As a consequence,

$$
\begin{gathered}
A \varphi+B \Gamma_{2}-C \Delta_{4}=A \varphi+B \Gamma_{2}-\left(O\left(\Delta_{2}\right)\right)^{2} \\
=A \varphi+B \Gamma_{2}-\left(O(\varphi)+O\left(\Gamma_{2}\right)\right)^{2}=A \varphi+B \Gamma_{2}-\left(O\left(\varphi^{2}\right)+O\left(\left(\Gamma_{2}\right)^{2}\right)\right) .
\end{gathered}
$$

Since both $\varphi(\tau)$ and $\Gamma_{2}(\tau)$ converge to 0 as $\tau$ converges to $b$, the terms $O\left(\varphi^{2}\right)+O\left(\left(\Gamma_{2}\right)^{2}\right)$ are of the order $o(\varphi)+o\left(\Gamma_{2}\right)$, and, choosing $\epsilon>0$ such that $\epsilon<A$ and $\epsilon<B$, there exists $\delta>0$ such that

$$
A \varphi(\tau)+B \Gamma_{2}(\tau)-C \Delta_{4}(\tau)>(A-\epsilon) \varphi(\tau)+(B-\epsilon) \Gamma_{2}(\tau)>0
$$

whenever $\tau \in U \cap \mathcal{H}$ and $|\tau-b|<\delta$, as desired.

Finally, we have

Proof of Theorem 1 completed. In the curvature formula (21) for bisectional curvatures the left-hand side $R_{\mu \bar{\mu} \alpha \bar{\alpha}}(\tau)$ is nonpositive, while by Lemma 1, the right-hand side is dominated by $\frac{1}{8 k}(q(q-1)-2) \varphi(\tau)$ as $\tau$ approaches a general boundary point $b \in \partial \mathcal{H}$. When $q \geq 3$ this dominant term is strictly positive, contradicting the nonpositivity of the left-hand side. The proof of Theorem 1 is complete.

\section{$\S 2$ Boundary behavior of asymptotically totally geodesic holomorphic isometries of the Poincaré disk}

(2.1) Holomorphic isometries of the Poincaré disk of the first kind In [Mk 4] we constructed examples of non-standard holomorphic isometric embeddings of the Poincaré disk into the polydisk. Here a holomorphic isometric embedding is said to be non-standard if and only if it fails to be totally geodesic. The simplest of such maps is the square root map, given in terms of unbounded realizations by the map $F: \mathcal{H} \rightarrow \mathcal{H} \times \mathcal{H}$ by $F(\tau)=(\sqrt{\tau}, i \sqrt{\tau})$, where $\mathcal{H}$ stands for the upper half-plane. In general, given any positive integer $p$, the $p$-th root map $F(\tau)=\left(e^{\frac{\pi i}{p}} \tau^{\frac{1}{p}}, \cdots, e^{\frac{(p-1) \pi i}{p}} \tau^{\frac{1}{p}}\right)$ is also a holomorphic isometry. Normalizing the Poincaré metric of the unit disk (or the upper half-plane) to be of constant Gaussian curvature -1, and doing the same for each of the individual Cartesian direct factor of the polydisk (or products 
of the upper half-plane), the holomorphic isometries given by the $p$-th root map are actually bona fide isometries. At any point $b \in \partial \mathcal{H}$ other than 0 , by direct calculation it is easily verified that the length $\|\sigma(\tau)\|$ at $F(\tau) \in \mathcal{H}^{p}$ of the second fundamental form $\sigma$ approaches to 0 as $\tau$ approaches $b$. As we will show, this turns out to be a general phenomenon for holomorphic isometries of the Poincaré disk into polydisks. To proceed we introduce first of all some terminology regarding holomorphic isometries of the Poincaré disk.

Definition 1. Let $\left(\Omega, d s_{\Omega}^{2}\right)$ be either an irreducible bounded symmetric domain or a Cartesian product of identical irreducible bounded symmetric domains, equipped with an Aut $(\Omega)$ invariant Kähler metric $d s_{\Omega}^{2}$ such that all minimal disks on $\Omega$ are of constant Gaussian curvature -1 . Let $d s_{\Delta}^{2}$ be the Poincaré metric on the unit disk $\Delta$ of constant Gaussian curvature -1 . Let $\lambda>0$ be a real number and $F:\left(\Delta, \lambda d s_{\Delta}^{2}\right) \rightarrow\left(\Omega, d s_{\Omega}^{2}\right)$ be a non-standard holomorphic isometry. Assume that $F$ is asymptotically totally geodesic at any boundary point $b \in \partial \Delta$ outside of a finite subset $E \subset \partial \Delta$. Let $\varphi$ be a real-analytic extension of the function $\|\sigma(\tau)\|^{2}$ to a neighborhood of $\Delta \cup(\partial \Delta-E)$. We say that

(a) $F:\left(\Delta, \lambda d s_{\Delta}^{2}\right) \rightarrow\left(\Omega, d s_{\Omega}^{2}\right)$ is a holomorphic isometry (or a holomorphic isometric embedding) of the first kind if and only if $\varphi$ vanishes to the order 2 at a general point of $\partial \Delta-E$;

(b) $F:\left(\Delta, \lambda d s_{\Delta}^{2}\right) \rightarrow\left(\Omega, d s_{\Omega}^{2}\right)$ is a holomorphic isometry (or a holomorphic isometric embedding) of the second kind if and only if $\varphi$ vanishes to the order 1 at a general point of $\partial \Delta-E$;

In what follows, to simplify the language, by a holomorphic isometry of the first resp. second kind of the Poincaré disk we will always mean a holomorphic isometry of the Poincaré disk into some $\left(\Omega, d s_{\Omega}^{2}\right)$ as described in (a) resp. (b) in Definition 1. In particular, it will be assumed implicitly that the holomorphic isometry is non-standard and that it is asymptotically totally geodesic at a general boundary point $b \in \partial \Delta$.

Theorem 2. On the unit disk $\Delta$ denote by $d s_{\Delta}^{2}$ the Poincaré metric of constant Gaussian curvature -1. In general, for $n \geq 1$ on the unit ball $B^{n}$ denote by $d s_{B^{n}}^{2}$ the canonical Kähler-Einstein metric of constant holomorphic sectional curvature -1 . Let $\lambda>0$ and $F:\left(\Delta, \lambda d s_{\Delta^{2}}^{2}\right) \rightarrow\left(B^{n}, d s_{B^{n}}^{2}\right) \times \cdots \times\left(B^{n}, d s_{B^{n}}^{2}\right)$ be a non-standard holomorphic isometry. Then, $F$ is asymptotically totally geodesic at a general boundary point $b \in \partial \mathcal{H}$. Moreover, $F$ is a holomorphic isometric embedding of the first kind of the Poincaré disk.

Proof. By means of the Cayley transform $\tau=\frac{z+i}{z-i}$ we will identify the disk with the upper half-plane $\mathcal{H}, d s_{\Delta}^{2}$ with $d s_{\mathcal{H}}^{2}$, and consider $F$ as being defined on the upper half-plane. Write $F(\tau)=\left(F_{1}(\tau), \cdots, F_{p}(\tau)\right)$, where each $F_{i}: \mathcal{H} \rightarrow B^{n}, 1 \leq k \leq p$, is a holomorphic map. We will call $F_{i}$ a component of $F$. At a general boundary point $b \in \partial \mathcal{H}, F$ and hence each component $F_{i}$ extend holomorphically to a neighborhood $U_{b}$ of $b$ in $\mathbb{C}$. Moreover, for each component $F_{i}$, the mapping $F_{i}$ defines a holomorphic embedding of $U_{b}$ onto a locally closed complex submanifold $S_{b} \subset \mathbb{C}^{n}$ and one of the following two alternatives occur: (a) $F\left(U_{b} \cap \partial \Delta\right) \subset \partial B^{n}$, and $d F_{i}\left(T_{b}(\mathbb{C})\right)$ is transversal to $\partial B^{n}$; or (b) $F_{i}(b)$ is an interior point, i.e., $F_{i}(b) \in B^{n}$. In what follows we make use of the coordinate $t=\operatorname{Im}(\tau)$. Write $\omega_{\mathcal{H}}$ for the Kähler form of $\left(\mathcal{H}, d s_{\mathcal{H}}^{2}\right)$, and $\omega_{B^{n}}$ for the Kähler form of $\left(B^{n}, d s_{B^{n}}^{2}\right)$. In a neighborhood $U_{b}$ 
of $b$ in $\mathbb{C}$, for an index $k, 1 \leq k \leq p$, such that Alternative (a) occurs, we have

$$
F_{i}^{*}\left(\omega_{B^{n}}\right)=\sqrt{-1} \partial \bar{\partial}\left(-2 \log \left(1-\left\|F_{i}\right\|^{2}\right)\right)
$$

Since $d F_{i}\left(T_{b}(\mathbb{C})\right)$ is transversal to $\partial B^{n}$, shrinking $U_{b}$ if necessary, the function $\rho:=1-\left\|F_{i}\right\|^{2}$, which is positive on $U_{b} \cap \mathcal{H}$ and vanishes on $U_{b} \cap \mathcal{H}$, satisfies $d \rho \neq 0$. Thus $-\rho$ is a local defining function on $U_{b} \cap \mathcal{H}$, and we have on $U_{b}$ the relation $\rho(\tau)=\mu(\tau) t$ for a some smooth positive function $\mu$ on $U_{b}$. Taking the complex Hessian of their logarithms we conclude that

$$
F_{i}^{*}\left(\omega_{B^{n}}\right)=\sqrt{-1} \partial \bar{\partial}(-2 \log t-2 \log \mu)=\omega_{\mathcal{H}}-2 \sqrt{-1} \partial \bar{\partial} \log \mu
$$

Since $\omega_{\mathcal{H}}=\frac{2}{t^{2}}(\sqrt{-1} d \tau \wedge d \bar{\tau})$ and $\log \mu$ is a smooth function on $U_{b}$, we conclude that

$$
F_{i}^{*}\left(\omega_{B^{n}}\right)=\left(1+O\left(t^{2}\right)\right) \omega_{\mathcal{H}}
$$

Now let $\tau \in \mathcal{H}$ converge to $b$. Denoting by $\eta=\eta(\tau)$ a unit vector at $\tau$ with respect to $d s_{\mathcal{H}}^{2}$, and writing $d F_{i}(\eta)=\left(\beta_{1}, \cdots \beta_{p}\right)$, we have

$$
\left\|\beta_{i}\right\|^{2}=1+a_{i} t^{2}+O\left(t^{3}\right) \quad \text { as } \quad \tau \rightarrow b
$$

for some constant $a_{i}$ whenever Alternative (a) holds true for the component $F_{i}$. Here from the Schwarz Lemma one can conclude that $a_{i} \leq 0$ but this is not used in the ensuing arguments. On the other hand, when Alternative (b) holds true for the component $F_{j}, F_{i}^{*}\left(\omega_{B^{n}}\right)$ is a smooth strictly positive $(1,1)$ form on a neighborhood of $b$, and we conclude that

$$
\left\|\beta_{j}\right\|^{2}=c_{j} t^{2}+O\left(t^{3}\right) \quad \text { as } \quad \tau \rightarrow b
$$

where each $c_{j}$ is strictly positive. Suppose there are $k$ components $F_{i}$ where Alternative (a) holds true and $p-k$ components $F_{j}$ where Alternative (b) holds true. From (4) and (5) we conclude that $\| d F\left(\alpha(\tau) \|^{2}\right.$ tends to $k$ as $\tau$ tends to $b$, so that the normalizing constant $\lambda$ in the statement of Theorem 2 must be a positive integer $k$. Clearly $1 \leq k \leq p$. If $k=p$ it follows readily from the Ahlfors-Schwarz Lemma that $F$ is totally geodesic. As a consequence, there is at least 1 component $F_{j}$ for which Alternative (b) holds true, so that $1 \leq k<p$. We have

$$
k=\|\beta\|^{2}=\sum\left(1+a_{i} t^{2}+O\left(t^{3}\right)\right)+\sum\left(c_{j} t^{2}+O\left(t^{3}\right)\right) .
$$

so that

$$
\sum a_{i}+\sum c_{j}=0, \quad \text { in particular } \sum c_{j}=-\sum a_{i}<0 .
$$

We proceed to compute curvatures. Since $\left(B^{n}, d s_{B^{n}}^{2}\right)$ is of constant holomorphic sectional curvature equal to -1 , writing $\alpha=\frac{1}{\sqrt{k}}\left(\beta_{1}, \cdots, \beta_{p}\right)$ and denoting by $R$ the curvature tensor of the target manifold, we have

$$
-R_{\alpha \bar{\alpha} \alpha \bar{\alpha}}=\frac{1}{k^{2}} \sum\left(1+a_{i} t^{2}+O\left(t^{3}\right)\right)^{2}+\sum\left(c_{j} t^{2}+O\left(t^{3}\right)\right)^{2}=\frac{1}{k}+\frac{4}{k^{2}}\left(\sum a_{i}\right) t^{2}+O\left(t^{3}\right),
$$


Since $F:\left(\mathcal{H}, k \cdot d s_{\mathcal{H}}\right) \rightarrow\left(B^{n}, d s_{B^{n}}^{2}\right) \times \cdots \times\left(B^{n}, d s_{B^{n}}^{2}\right)$ is a holomorphic isometry, the embedded (closed) complex submanifold $S:=F(\mathcal{H}) \subset B^{n} \times \cdots \times B^{n}$ is of constant Gaussian curvature $-\frac{1}{k}$. Recall from the structural equation [(1.2), Eqn.(1)], we have $-R_{\alpha \bar{\alpha} \alpha \bar{\alpha}}=\frac{1}{k}+\|\sigma\|^{2}$ for the second fundamental form $\sigma$ of the holomorphic embedding $F$. It follows from (8) that

$$
\|\sigma\|^{2}=\frac{4}{k^{2}}\left(-\sum a_{i}\right) t^{2}+O\left(t^{3}\right) .
$$

We conclude first of all that $F$ is asymptotically totally geodesic at a general boundary point $b \in \partial \mathcal{H}$. Since $\sum a_{i}<0$ by $(7)$, we conclude that $\varphi=\|\sigma\|^{2}$ vanishes precisely to the order 2 at a general boundary point. Hence, the holomorphic isometry $F$ is of the first kind, and the proof of Theorem 2 is complete.

(2.2) An example of a holomorphic isometry of the Poincaré disk into a Siegel upper halfplane We constructed in $[\mathrm{Mk} 4]$ an example of a proper holomorphic isometric embedding from the Poincaré disk into some Siegel upper half-plane which does not arise from the $p$-root map. More precisely we have

Proposition (Mok[Mk4, Proposition 2.3.2]). For $\tau \in \mathbb{C}, \tau=\rho e^{i \varphi}, \rho>0,-\pi<\varphi<\pi$, and for any positive integer we write $\tau^{\frac{1}{n}}=\rho^{\frac{1}{n}} e^{\frac{i \varphi}{n}}$. Write $M_{s}(n, \mathbb{C})$ for the vector space of n-by-n symmetric matrices with complex coefficients. Then, the holomorphic mapping $F: \mathcal{H} \rightarrow M_{s}(6, \mathbb{C})$ defined by

$$
F(\tau)=\left[\begin{array}{cccccc}
i \tau^{\frac{1}{3}} & \sqrt{2} \tau^{\frac{1}{6}} & 0 & 0 & 0 & 0 \\
\sqrt{2} \tau^{\frac{1}{6}} & i & 0 & 0 & 0 & 0 \\
0 & 0 & i \tau^{\frac{1}{6}} & 0 & 0 & 0 \\
0 & 0 & 0 & i(-\tau)^{\frac{1}{3}} & \sqrt{2}(-\tau)^{\frac{1}{6}} & 0 \\
0 & 0 & 0 & \sqrt{2}(-\tau)^{\frac{1}{6}} & i & 0 \\
0 & 0 & 0 & 0 & 0 & i(-\tau)^{\frac{1}{6}}
\end{array}\right]
$$

maps $\mathcal{H}$ into $\mathcal{H}_{6}$, and it is a proper holomorphic isometric embedding with respect to the Poincaré metric $d s_{\mathcal{H}}^{2}$ of constant Gaussian curvature -1 on $\mathcal{H}$ and the normalized invariant Kähler metric $d s_{\mathcal{H}_{6}}^{2}$ on $\mathcal{H}_{6}$ of minimal holomorphic sectional curvature -1 .

In the rest of the article we examine specifically this non-standard holomorphic isometry of the Poincaré disk. We prove

Proposition 2. The holomorphic map $F:\left(\mathcal{H}, d s_{\mathcal{H}}^{2}\right) \rightarrow\left(\mathcal{H}_{6}, d s_{\mathcal{H}_{6}}^{2}\right)$ as described in the above is a holomorphic isometric embedding of the first kind.

Proof. Let $(-\infty, 0] \subset \mathbb{R}$ be the nonpositive part of the real axis. Write $F(\tau)=\left[\begin{array}{cc}H(\tau) & 0 \\ 0 & H(-\tau)\end{array}\right]$, where $H: \mathbb{C}-(-\infty, 0] \rightarrow M_{s}(3, \mathbb{C})$. Define also $G: \mathbb{C}-(-\infty, 0] \rightarrow M_{s}(2, \mathbb{C})$ by $G(\tau)=$ $\left[\begin{array}{cc}i \tau^{\frac{1}{3}} & \sqrt{2} \tau^{\frac{1}{6}} \\ \sqrt{2} \tau^{\frac{1}{6}} & i\end{array}\right]$. Thus, we have

$$
H(\tau)=\left[\begin{array}{cc}
G(\tau) & 0 \\
0 & i \tau^{\frac{1}{6}}
\end{array}\right] \quad \text { and } \quad F(\tau)=\left[\begin{array}{cccc}
G(\tau) & 0 & 0 & 0 \\
0 & i \tau^{\frac{1}{6}} & 0 & 0 \\
0 & 0 & G(-\tau) & 0 \\
0 & 0 & 0 & i(-\tau)^{\frac{1}{6}}
\end{array}\right]
$$


We will also write $H(\tau)=\operatorname{Diag}\left(G(\tau), i \tau^{\frac{1}{6}}\right)$ and $F(\tau)=\operatorname{Diag}\left(G(\tau), i \tau^{\frac{1}{6}}, G(-\tau), i(-\tau)^{\frac{1}{6}}\right)$, as short-hand notations. To compute the second fundamental form, consider $\beta=d F\left(\frac{\partial}{\partial \tau}\right)$ and $\alpha=\frac{\beta}{\|\beta\|}$. Then, from the structural equation we have

$$
R_{\alpha \bar{\alpha} \alpha \bar{\alpha}}=\|\sigma\|^{2}-1 \text {. }
$$

At the standard reference point $i I \in \mathcal{H}_{6}$, the canonical Kähler-Einstein metric $d s_{\mathcal{H}_{6}}^{2}$, normalized so that minimal disks are of constant Gaussian curvature -1, is the same as one half of the Euclidean metric at iI (cf. Siegel [Si,Theorem 3, p.129]) To compute the length of the $(1,0)$-vector $\beta$ at $F(\tau)$ with respect to $d s_{\mathcal{H}_{6}}^{2}$ it suffices to transform $\beta$ to a $(1,0)$ vector by means of an automorphism of $\mathcal{H}_{6}$. Using the standard trivialization of the holomorphic tangent bundle of the Euclidean space in terms of Euclidean coordinates, identify $\beta=d F\left(\frac{\partial}{\partial \tau}\right)=F^{\prime}(\tau)$ with a matrix $M(\tau)$ at $F(\tau)$. Now $W(\tau):=\operatorname{Im}(F(\tau))>0$. In what follows for a real positive definite matrix $P$ we will denote by $P^{\frac{1}{2}}$ its unique positive definite square root, and by $P^{-\frac{1}{2}}$ its multiplicative inverse, which is also positive definite. The linear transformation $\Lambda_{\tau}(Z):=W(\tau)^{-\frac{1}{2}} Z W(\tau)^{-\frac{1}{2}}$ is an automorphism of $\mathcal{H}_{6}$. We have $\Lambda_{\tau}(F(\tau))=W(\tau)^{-\frac{1}{2}} F(\tau) W(\tau)^{-\frac{1}{2}}$, so that $\operatorname{Im}\left(\Lambda_{\tau}(F(\tau))=W(\tau)^{-\frac{1}{2}} W(\tau) W(\tau)^{-\frac{1}{2}}=I\right.$. $d \Lambda_{\tau}\left(d F\left(\frac{\partial}{\partial \tau}\right)\right)=d \Lambda_{\tau}(\beta)$ is identified with the matrix $W(\tau)^{-\frac{1}{2}} M(\tau) W(\tau)^{-\frac{1}{2}}$. Note that for an $n$-by- $m$ matrix $Q$ with complex coefficients, the square of its Euclidean norm is the same as $\operatorname{Tr}\left(Q \overline{Q^{t}}\right)$, where $Q^{t}$ denotes the transpose of $Q$. Since a translation of the form $T(Z)=Z+N$ for a real symmetric matrix $N$ is an automorphism on the Siegel upper half-plane, thus preserving the canonical Kähler-Einstein metric, the automorphism $Z \mapsto \Lambda_{\tau}(Z)-\operatorname{Re}(F(\tau))$ transforms $F(\tau)$ to $i I$, and we have

$$
\begin{gathered}
\|\beta\|^{2}=\left\|d \Lambda_{\tau}(\beta)\right\|^{2}=\frac{1}{2}\left\|W^{-\frac{1}{2}} M W^{-\frac{1}{2}}\right\|_{\text {Euclid }}^{2} \\
\left.=\frac{1}{2} \operatorname{Tr}\left(W^{-\frac{1}{2}} M W^{-\frac{1}{2}}\right)\left(W^{-\frac{1}{2}} \bar{M} W^{-\frac{1}{2}}\right)\right)=\frac{1}{2} \operatorname{Tr}\left(W^{-\frac{1}{2}} M W^{-1} \bar{M} W^{-\frac{1}{2}}\right),
\end{gathered}
$$

noting in the second last equality that $\overline{\left(W^{-\frac{1}{2}} M W^{-\frac{1}{2}}\right)^{t}}=W^{-\frac{1}{2}} \bar{M} W^{-\frac{1}{2}}$ since both $W^{-\frac{1}{2}}$ and $M$ are symmetric, and $W^{-\frac{1}{2}}$ is real. Here and in what follows $\|\cdot\|$ denotes the norm with respect to the canonical metric $d s_{\mathcal{H}_{n}}^{2}$ on the Siegel upper half-plane, $\|\cdot\|_{\text {Euclid }}$ the norm with respect to the Euclidean metric, and $W$ is a short-hand for $W(\tau)$, etc. Recall that for a square $n$-by- $n$ matrix $Q$ and an invertible $n$-by- $n$ matrix $P$, we have $\operatorname{Tr}\left(P Q P^{-1}\right)=\operatorname{Tr}(Q)$. Applying this to (3) by taking $P$ to be $W^{\frac{1}{2}}$, and denoting by $E$ the (not necessarily symmetric) matrix $M W^{-1}$ we have

$$
\|\beta\|^{2}=\frac{1}{2} \operatorname{Tr}\left(W^{\frac{1}{2}}\left(W^{-\frac{1}{2}} M W^{-1} \bar{M} W^{-\frac{1}{2}}\right) W^{\frac{1}{2}}\right)=\frac{1}{2} \operatorname{Tr}\left(M W^{-1} \overline{M W^{-1}}\right)=\frac{1}{2} \operatorname{Tr}(E \bar{E}) .
$$

The formula (4) is the same as that given in Siegel [Si,Theorem 3, p.129] except for a different normalizing constant, noting that $\operatorname{Tr}\left(M W^{-1} \overline{M W^{-1}}\right)=\operatorname{Tr}\left(W^{-1} \overline{M W^{-1} M}\right)=\operatorname{Tr}(D \bar{D})$, where $D=W^{-1} M$, which was used in [Si, loc. cit.]. For a $(1,0)$ vector at the reference point $i I$ represented by $X \in M_{s}(6 ; \mathbb{C})$ the holomorphic sectional curvature with respect to $d s_{\mathcal{H}_{6}}^{2}$ is on the other hand determined by

$$
-R_{X \bar{X} X \bar{X}}=\frac{1}{4}\|X \bar{X}\|^{2}=\frac{1}{4} \operatorname{Tr}(X \bar{X} X \bar{X}) .
$$


Here the curvature formula can be deduced by the formula at the origin for the HarishChandra realization of $\mathcal{H}_{n}$ as a classical domain $D_{n}^{I I I}$ of type $I I I, D_{n}^{I I I}=\left\{Z \in M_{s}(n, \mathbb{C})\right.$ : $I-\bar{Z} Z>0\} . D_{n}^{I I I}$ is a totally geodesic complex submanifold of a domain $D_{n, n}^{I}$ of type I, and the curvature for the latter at 0 , at which the Euclidean coordinates are complex geodesic coordinates, is computed in Mok ([M2], (4.3), p.84). iI $\in \mathcal{H}_{n}$ corresponds to $0 \in D_{n}^{I I I}$ under the inverse Cayley transform $Z=(\tau-i I)(\tau+i I)^{-1}$, and a direct computation shows that the Jacobian matrix at $\tau=i I$ is a multiple of the identity. The constant $\frac{1}{4}$ appearing in (5) can be determined from the case $n=1$, where $\left\|\frac{\partial}{\partial \tau}\right\|^{4}=\frac{1}{4}$ and the Gaussian curvature is -1 . Recall that $F(\tau)=\operatorname{Diag}\left(G(\tau), i \tau^{\frac{1}{6}}, G(-\tau), i(-\tau)^{\frac{1}{6}}\right), E(\tau)=M(\tau) W^{-1}(\tau)=F^{\prime}(\tau)(\operatorname{Im} F(\tau))^{-1}$. $F(\tau)$ is defined at each $\tau_{0} \in(-\infty, 0)$ and holomorphic on some neigborhood $U$ of $\tau_{0}$. For $\tau_{0} \in(-\infty, 0)$ we have

$$
\begin{aligned}
\operatorname{Im} F\left(\tau_{0}\right)= & \operatorname{Diag}\left(\operatorname{Im} G\left(\tau_{0}\right), \operatorname{Im}\left(i\left|\tau_{0}\right|^{\frac{1}{6}} e^{\frac{\pi i}{6}}\right), \operatorname{Im} G\left(-\tau_{0}\right), \operatorname{Im}\left(i\left|\tau_{0}\right|^{\frac{1}{6}} e^{-\frac{\pi i}{6}}\right)\right) \\
& =\left(\operatorname{Im} G\left(\tau_{0}\right), \frac{1}{2}\left|\tau_{0}\right|^{\frac{1}{6}}, \operatorname{Im} G\left(-\tau_{0}\right),-\frac{1}{2}\left|\tau_{0}\right|^{\frac{1}{6}}\right)
\end{aligned}
$$

Write $a(\tau)=i \tau^{\frac{1}{6}}, b(\tau)=i(-\tau)^{\frac{1}{6}}$. Note that $a\left(\tau_{0}\right) \in \mathcal{H}$ and $b\left(\tau_{0}\right) \in \mathcal{H}$ for any $\tau_{0} \in(-\infty, 0)$, i.e. they are both interior points. Moreover

$$
\operatorname{Im} G\left(\tau_{0}\right)=\operatorname{Im}\left[\begin{array}{cc}
i\left|\tau_{0}\right|^{\frac{1}{3}} & \sqrt{2}\left|\tau_{0}\right|^{\frac{1}{6}} \\
\sqrt{2}\left|\tau_{0}\right|^{\frac{1}{6}} & i
\end{array}\right]=\left[\begin{array}{cc}
\left|\tau_{0}\right|^{\frac{1}{3}} & 0 \\
0 & 1
\end{array}\right]>0
$$

so that $G\left(-\tau_{0}\right) \in \mathcal{H}_{2}$, i.e., it is an interior point on the Siegel upper left-plane $\mathcal{H}_{2}$. Now

$$
\left.E(\tau)=\operatorname{Diag}\left(G^{\prime}(\tau)(\operatorname{Im} G(\tau))^{-1}, \frac{a^{\prime}(\tau)}{\operatorname{Im} a(\tau)},-G^{\prime}(-\tau)(\operatorname{Im} G(-\tau))^{-1}\right), \frac{b^{\prime}(\tau)}{\operatorname{Im} G(\tau)}\right)
$$

As $\tau \in \mathcal{H}$ approaches $\tau_{0} \in(-\infty, 0)$, the scalar functions $\frac{a^{\prime}(\tau)}{\operatorname{Im} a(\tau)}$ and $\frac{b^{\prime}(\tau)}{\operatorname{Im} b(\tau)}$ are bounded, and the matrix-valued function $-G^{\prime}(-\tau)(\operatorname{Im} G(-\tau))^{-1}$ is also bounded. In fact, all the functions concerned extend real-analytically to a neighborhood of $\tau_{0}$ in $\mathbb{C}$. If we write $Y(\tau)=\operatorname{Im} G(\tau)$ and $A(\tau)=G^{\prime}(\tau) Y(\tau)^{-1}$, from the fact that $F: \mathcal{H} \rightarrow \mathcal{H}_{6}$ is an isometry it follows that

$$
\frac{1}{2 t^{2}}=\left\|d f\left(\frac{\partial}{\partial \tau}\right)\right\|^{2}=\frac{1}{2} \operatorname{Tr}(E(\tau) \overline{E(\tau)})=\frac{1}{2} \operatorname{Tr}(A(\tau) \overline{A(\tau)})+O(1)
$$

For the computation of curvature note that

$$
X(\tau)=\operatorname{Diag}\left(Y(\tau)^{-\frac{1}{2}} G^{\prime}(\tau) Y(\tau)^{-\frac{1}{2}}, \frac{a^{\prime}(\tau)}{\operatorname{Im} a(\tau)},-Y(\tau)^{-\frac{1}{2}} G^{\prime}(-\tau) Y(\tau)^{-\frac{1}{2}}, \frac{b^{\prime}(\tau)}{\operatorname{Im} G(\tau)}\right)
$$

Write $S(\tau)=Y(\tau)^{-\frac{1}{2}} G^{\prime}(\tau) Y(\tau)^{-\frac{1}{2}}$. Noting that $Y(-\tau)^{-\frac{1}{2}}$ and $G^{\prime}(-\tau)$ are defined and finite at $\tau=\tau_{0} \in(-\infty, 0)$ we have as in (9) the relation

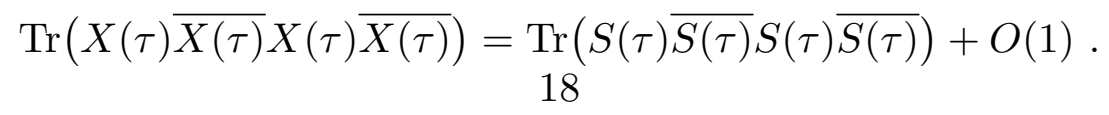


Thus, for $\tau \in \mathcal{H}, \beta=d F\left(\frac{\partial}{\partial \tau}\right), \alpha=\frac{\beta}{\|\beta\|}$, we have

$$
-R_{\alpha \bar{\alpha} \alpha \bar{\alpha}}=\frac{\operatorname{Tr}(X(\tau) \overline{X(\tau)} X(\tau) \overline{X(\tau)})}{(\operatorname{Tr}(X(\tau) \overline{X(\tau)}))^{2}}=\frac{\operatorname{Tr}(S(\tau) \overline{S(\tau)} S(\tau) \overline{S(\tau)})}{(\operatorname{Tr}(S(\tau) \overline{S(\tau)}))^{2}}+O\left(t^{2}\right)
$$

To prove that $F$ is asymptotically geodesic at $\tau_{0} \in(-\infty, 0)$ and further that it is of the first kind it suffices therefore to show that

$$
\text { (†) } \quad \frac{\operatorname{Tr}(S(\tau) \overline{S(\tau)} S(\tau) \overline{S(\tau)})}{(\operatorname{Tr}(S(\tau) \overline{S(\tau)}))^{2}}=1+O\left(t^{2}\right)
$$

To prove $(\dagger)$ we proceed to compute in terms of $A=A(\tau)$, recalling first of all that $\operatorname{Tr}(A \bar{A})=$ $\operatorname{Tr}(S \bar{S})$. We have

$$
\begin{gathered}
G^{\prime}=\left[\begin{array}{cc}
\frac{i}{3} \tau^{-\frac{2}{3}} & \frac{\sqrt{2}}{6} \tau^{-\frac{5}{6}} \\
\frac{\sqrt{2}}{6} \tau^{-\frac{5}{6}} & 0
\end{array}\right]=\tau^{-\frac{5}{6}}\left[\begin{array}{cc}
\frac{i}{3} \tau^{\frac{1}{6}} & \frac{\sqrt{2}}{6} \\
\frac{\sqrt{2}}{6} & 0
\end{array}\right] \\
Y=\operatorname{Im}\left[\begin{array}{cc}
i \tau^{\frac{i}{3}} & \sqrt{2} \tau^{\frac{1}{6}} \\
\sqrt{2} \tau^{\frac{1}{6}} & i
\end{array}\right]=\left[\begin{array}{cc}
\operatorname{Re} \tau^{\frac{1}{3}} & \sqrt{2} \operatorname{Im} \tau^{\frac{1}{6}} \\
\sqrt{2} \operatorname{Im} \tau^{\frac{1}{6}} & 1
\end{array}\right] .
\end{gathered}
$$

Write $\tau^{\frac{1}{6}}=\lambda+i \mu$. Then $\tau^{\frac{1}{3}}=(\lambda+i \mu)^{2}=\left(\lambda^{2}-\mu^{2}\right)+2 i \lambda \mu$, and

$$
Y=\left[\begin{array}{cc}
\lambda^{2}-\mu^{2} & \sqrt{2} \mu \\
\sqrt{2} \mu & 1
\end{array}\right]
$$

Thus,

$$
\begin{gathered}
Y^{-1}=\frac{1}{\lambda^{2}-3 \mu^{2}}\left[\begin{array}{cc}
1 & -\sqrt{2} \mu \\
-\sqrt{2} \mu & \lambda^{2}-\mu^{2}
\end{array}\right] ; \\
A=G^{\prime} Y^{-1}=\frac{\tau^{-\frac{5}{6}}}{\lambda^{2}-3 \mu^{2}}\left[\begin{array}{cc}
\frac{i}{3} \tau^{\frac{1}{6}} & \frac{\sqrt{2}}{6} \\
\frac{\sqrt{2}}{6} & 0
\end{array}\right]\left[\begin{array}{cc}
1 & -\sqrt{2} \mu \\
-\sqrt{2} \mu & \lambda^{2}-\mu^{2}
\end{array}\right] \\
=\frac{\tau^{-\frac{5}{6}}}{6\left(\lambda^{2}-3 \mu^{2}\right)}\left[\begin{array}{cc}
2 i(\lambda+i \mu) & \sqrt{2} \\
\sqrt{2} & 0
\end{array}\right]\left[\begin{array}{cc}
1 & -\sqrt{2} \mu \\
-\sqrt{2} \mu & \lambda^{2}-\mu^{2}
\end{array}\right] \\
=\frac{\tau^{-\frac{5}{6}}}{6\left(\lambda^{2}-3 \mu^{2}\right)}\left[\begin{array}{ccc}
-4 \mu+2 i \lambda & 2 \sqrt{2}\left(\mu^{2}-i \lambda \mu\right)+\sqrt{2}\left(\lambda^{2}-\mu^{2}\right) \\
\sqrt{2} & -2 \mu
\end{array}\right]:=\frac{\tau^{-\frac{5}{6}}}{6\left(\lambda^{2}-3 \mu^{2}\right)} A_{0}, \\
=\frac{\tau^{-\frac{5}{6}}}{6\left(\lambda^{2}-3 \mu^{2}\right)}\left[\begin{array}{cc}
-4 \mu+2 i \lambda \\
\sqrt{2}\left(\lambda^{2}-3 \mu^{2}\right)+4 \sqrt{2} \mu^{2}-2 \sqrt{2} i \lambda \mu
\end{array}\right]
\end{gathered}
$$


For $\tau_{0} \in(-\infty, 0)$, we have $\lambda+i \mu=\tau_{0}^{\frac{1}{6}}=\left|\tau_{0}\right|^{\frac{1}{6}} e^{\frac{\pi i}{6}}$, so that $\lambda=\tan \left(\frac{\pi i}{6}\right) \mu=\sqrt{3} \mu$, hence $\lambda^{2}-3 \mu^{2}=0$. Thus for $\tau_{0} \in(-\infty, 0)$ we have

$$
\begin{gathered}
A_{0}=\left[\begin{array}{cc}
(-4+2 \sqrt{3} i) \mu & (4 \sqrt{2}-2 \sqrt{6} i) \mu^{2} \\
\sqrt{2} & -2 \mu
\end{array}\right] \\
A_{0} \bar{A}_{0}=\left[\begin{array}{cc}
(-4+2 \sqrt{3} i) \mu & (4 \sqrt{2}-2 \sqrt{6} i) \mu^{2} \\
\sqrt{2} & -2 \mu
\end{array}\right]\left[\begin{array}{cc}
(-4-2 \sqrt{3} i) \mu & (4 \sqrt{2}+2 \sqrt{6} i) \mu^{2} \\
\sqrt{2} & -2 \mu
\end{array}\right] \\
=\left[\begin{array}{cc}
(36-4 \sqrt{3} i) \mu^{2} & (-36 \sqrt{2}+4 \sqrt{6} i) \mu^{3} \\
(-6 \sqrt{2}-2 \sqrt{6} i) \mu & (12+4 \sqrt{3} i) \mu^{2}
\end{array}\right] \\
\operatorname{Tr}\left(A_{0} \bar{A}_{0}\right)=48 \mu^{2} \neq 0
\end{gathered}
$$

Thus in a neighborhood $U$ of $\tau_{0} \in(-\infty, 0), \operatorname{Tr}\left(A_{0} \bar{A}_{0}\right):=p(\tau)$ is a continuous strictly positive function. Note that in (18), the two column vectors of $A_{0}$ are proportional to each other over $(-\infty, 0)$. It follows that $\operatorname{det}\left(A_{0}\right)=0$ on $(-\infty, 0)$, while for $\tau \in \mathcal{H}$ we obtain from $(17)$

$$
\operatorname{det}\left(A_{0}\right)=-2\left(\lambda^{2}-3 \mu^{2}\right)
$$

Obviously the vanishing order of $\lambda^{2}-3 \mu^{2}=(\lambda-\sqrt{3} \mu)(\lambda+\sqrt{3} \mu)$ along the negative real axis is precisely equal to 1 , so that

$$
\operatorname{det}\left(A_{0} \overline{A_{0}}\right)=\left|\operatorname{det}\left(A_{0}\right)\right|^{2}=4\left(\lambda^{2}-3 \mu^{2}\right)^{2}=q(\tau) t^{2},
$$

for some continuous strictly positive function $q(\tau)$ defined on $U$. Starting from (20) and (22) we proceed to verify $(\dagger)$. Suppose the normal form of $\nu=\frac{G^{\prime}(\tau)}{\left\|G^{\prime}(\tau)\right\|}$ at $G(\tau)$ is given by $\operatorname{Diag}\left(\nu_{1}(\tau), \nu_{2}(\tau)\right)$, where $\nu_{1}(\tau)$ and $\nu_{2}(\tau)$ are nonnegative numbers and $\nu_{1} \geq \nu_{2}$. Then, $\nu_{1}^{2}+\nu_{2}^{2}=1$. Regarding $S$ as a tangent vector at the reference point $i I \in \mathcal{H}, \nu_{1}^{2}$ and $\nu_{2}^{2}$ are the eigenvalues of the Hermitian matrix $\frac{S \bar{S}}{\operatorname{Tr}(S \bar{S})}$. (Note here that $S \bar{S}$ is Hermitian symmetric since $S$ is complex symmetric, while $A \bar{A}$ is not a priori Hermitian symmetric.) From the curvature formula (5) we have

$$
R_{\nu \bar{\nu} \nu \bar{\nu}}=-\frac{\operatorname{Tr}(S \bar{S} S \bar{S})}{(\operatorname{Tr}(S \bar{S}))^{2}}=-\left(\nu_{1}^{4}+\nu_{2}^{4}\right) .
$$

On the other hand, we have also

$$
\operatorname{det}(S \bar{S})=|\operatorname{det}(S)|^{2}=\left|\operatorname{det}\left(Y^{-\frac{1}{2}} G^{\prime} Y^{-\frac{1}{2}}\right)\right|^{2}=\left|\operatorname{det}\left(G^{\prime} Y^{-1}\right)\right|^{2}=|\operatorname{det}(A)|^{2}=\operatorname{det}(A \bar{A}) .
$$

As a consequence,

$$
\nu_{1}^{2} \nu_{2}^{2}=\frac{\operatorname{det}(S \bar{S})}{\operatorname{Tr}(S \bar{S}))^{2}}=\frac{\operatorname{det}(A \bar{A})}{(\operatorname{Tr}(A \bar{A}))^{2}}=\frac{\operatorname{det}\left(A_{0} \bar{A}_{0}\right)}{\left(\operatorname{Tr}\left(A_{0} \bar{A}_{0}\right)\right)^{2}}=\frac{q(\tau) t^{2}}{p(\tau)^{2}} .
$$


where in the third equality we make use of the fact that $A(\tau)=h(\tau) A_{0}(\tau)$ for some scalar function $h(\tau)$ for $\tau \in U \cap \mathcal{H}$, so that $\operatorname{det}(A \bar{A})=h^{2} \operatorname{det}\left(A_{0} \bar{A}_{0}\right)$ and also $\left(\operatorname{Tr}\left(A_{0} \bar{A}_{0}\right)\right)^{2}=$ $h^{2}\left(\operatorname{Tr}\left(A_{0} \bar{A}_{0}\right)\right)^{2}$. Since $\frac{1}{2} \leq \nu_{1}^{2} \leq 1$ we conclude that there must exist positive numbers $c_{1}$ and $c_{2}$ such that $c_{1} t \leq \nu_{2}(\tau) \leq c_{2} t$ on $U \cap \mathcal{H}$, shrinking the neighborhood $U$ of $b$ if necessary. In particular, $\nu_{2}^{2}=O\left(t^{2}\right)$. Since $-R_{\nu \bar{\nu} \nu \bar{\nu}}=\nu_{1}^{4}+\nu_{2}^{4}=1-2 \nu_{1}^{2} \nu_{2}^{2}=1+O\left(t^{2}\right)$, by (23) we have established $(\dagger)$. The proof of Proposition 2 is complete.

Remarks. The proof of Proposition 2 actually shows without using Theorem 1 that for the example $F:\left(\mathcal{H}, d s_{\mathcal{H}}^{2}\right) \rightarrow\left(\mathcal{H}_{6}, d s_{\mathcal{H}}^{2}\right)$, the square of the norm of the second fundamental form $\varphi=\|\sigma\|^{2}$ actually vanishes along $\partial \mathcal{H}-\{0\}$ exactly to the order 2 . In the terminology of (1.4), for any point $\tau_{0} \in \partial \mathcal{H}-\{0\}$, there is exactly 1 stretching principal direction and 5 contracting principal directions. At a general point $\tau_{0} \in \partial \mathcal{H}-\{0\}$, the associated invariants $\gamma_{1} ; \delta_{1}, \cdots, \delta_{5}$ are given by $\delta_{j}(\tau)=d_{j}(\tau) t$, where $d_{j}(\tau)$ are germs of continuous positive functions at $\tau_{0}$ and by $\gamma_{1}(\tau)=1-c(\tau) t^{2}$, where $c$ is a germ of continuous positive function at $\tau_{0}$. It follows readily that $-R_{\alpha \bar{\alpha} \alpha \bar{\alpha}}=1-4 c(\tau) t^{2}+O\left(t^{4}\right)$, which implies that $\varphi=\|\sigma\|^{2}$ vanishes exactly to the order 2 at $\tau_{0}$, i.e., $F$ is a non-standard holomorphic isometry of the first kind.

(2.3) Open questions In this article we have been discussing about holomorphic isometries of the Poincaré disk into bounded symmetric domains $\Omega$, where for simplicity $\Omega$ is assumed to be either irreducible or a Cartesian product of identical irreducible bounded symmetric domains. In a forthcoming article we will show that in this context any holomorphic isometry of the Poincaré disk is necessarily asymptotically totally geodesic. Thus, any non-standard holomorphic isometric embedding of the Poincaré disk into $\Omega$ must necessarily be of the first kind or of the second kind. Our discussion in (2.1) and (2.2) reveals that, from the basic examples in Mok [Mk4], together with the standard constructions including the reparametrization of the Poincaré disk and branching of disk factors in the case of holomorphic isometries into polydisks, all known examples of non-standard holomorphic isometries into $\Omega$ are necessarily of the first kind. One is therefore naturally led to the following question.

Question 1 Does there exist a bounded symmetric domain $\Omega$ which is either irreducible or a Cartesian product of identical irreducible bounded symmetric domains, a positive constant $\lambda$ and a non-standard holomorphic isometry of the Poincaré disk $F:\left(\Delta, \lambda d s_{\Delta}^{2}\right) \rightarrow\left(\Omega, d s_{\Omega}^{2}\right)$ of the second kind?

Another feature of the known examples of non-standard holomorphic isometries of the Poincaré disk in our context is the fact that they always develop singularities along the unit circle, leading us naturally to the following question.

Question 2 Does there exist a bounded symmetric domain $\Omega$ which is either irreducible or a Cartesian product of identical irreducible bounded symmetric domains, a positive constant $\lambda$ and a non-standard holomorphic isometry of the Poincaré disk $F:\left(\Delta, \lambda d s_{\Delta}^{2}\right) \rightarrow\left(\Omega, d s_{\Omega}^{2}\right)$ such that $F$ extends holomorphically to a neighborhood $D$ of the closed unit disk $\bar{\Delta}$.

Questions 1 and 2 are probably interrelated, for the following reason. In the case of holomorphic isometries of the Poincaré disk into polydisks, the study of asymptotic behavior of holomorphic isometries leads to a differential equation on the germ along the unit circle of the square of the norm of the second fundamental form, which in turn implies that all such 
non-standard holomorphic isometries of the Poincaré disk into $D$ must develop singularities along the unit circle, as is the case of the $p$-th root map (Mok [Mk5]). As is proven in $[(2.1)$, Theorem 2] all holomorphic isometries of the Poincaré disk into polydisks are necessarily of the first kind. It is conceivable that the existence of singularities along the boundary circle already follows whenever the non-standard holomorphic isometry is of the first kind, irrespective of the target bounded symmetric domain $\Omega$. Granting this, an example of a non-standard holomorphic isometry of the Poincaré disk which extends to a neighborhood of the closed unit disk is necessarily a holomorphic isometry of the second kind. In relation to the existence problem in Question 1, while the evidence against existence provided by (2.1) and (2.2) of the current article is not overwhelming, the author is inclined to believe that the answer is negative. Hence conceivably the answer to Question 2 is also negative modulo some further arguments. This would have the interesting potential consequence that non-standard holomorphic isometries can be studied in terms of branch points developed on the unit circle and their images on the boundaries of bounded symmetric domains.

\section{References}

[Ca] Calabi, E.: Isometric imbedding of complex manifolds, Ann. Math. 58 (1953), 1-23.

[CU] Clozel, Laurent and Ullmo, E.: Modular correspondences and invariant measures, $J$. Reine Angew. Math. 558 (2003), 47-83.

[Mk1] Mok, N.: Uniqueness theorems of Hermitian metrics of seminegative curvature on locally symmetric spaces of negative Ricci curvature, Ann. Math. 125 (1987), 105-152.

[Mk2] Mok, N.: Metric Rigidity Theorems on Hermitian Locally Symmetric Manifolds, Series in Pure Mathematics Vol.6, World Scientific, Singapore-New Jersey-London-Hong Kong, 1989.

[Mk3] Mok, N.: Local holomorphic isometric embeddings arising from correspondences in the rank-1 case, in Contemporary Trends in Algebraic Geometry and Algebraic Topology, ed. S.-S. Chern, L. Fu and R. Hain, Nankai Tracts in Mathematics, Vol.5, World Scientific, New Jersey 2002, pp.155-166.

[Mk4] Mok, N.: Extension of germs of holomorphic isometries up to normalizing constants with respect to the Bergman metric. Preprint.

[Mk5] Mok, N.: Characterization of totally geodesic embeddings among holomorphic isometries of the Poincaré disk into a Cartesian product of complex unit balls. In preparation.

[Ng] Ng, S.C.: On holomorphic isometric embeddings from the unit disk into polydisks and their generalizations. Thesis at the University of Hong Kong, 2008.

[Si] Siegel, C. L. Topics in Complex Function Theory, Volume III, Abelian Functions and Modular Functions of Several Variables, John Wiley \& Sons Inc., New York-LondonSydney-Toronto, 1973.

[Xu] Xu, Y.: Theory of Complex Homogeneous Bounded Domains, Sciences Press, BeijingNew York \& Kluwer Academic Publishers, Dordrecht-Boston-London 2005.

[Wo] Wolf, J. A. Fine structure of Hermitian symmetric spaces, in Geometry of Symmetric Spaces, ed. Boothby-Weiss, Marcel-Dekker, New York, 1972, pp.271-357. 
Ngaiming Mok, The University of Hong Kong, Pokfulam Road, Hong Kong

(E-mail: nmok@hkucc.hku.hk) 